MATHEMATICS OF COMPUTATION

Volume 76, Number 260, October 2007, Pages 1743-1769

S 0025-5718(07)01965-5

Article electronically published on May 3, 2007

\title{
A DUAL FINITE ELEMENT COMPLEX ON THE BARYCENTRIC REFINEMENT
}

\author{
ANNALISA BUFFA AND SNORRE H. CHRISTIANSEN
}

\begin{abstract}
Given a two dimensional oriented surface equipped with a simplicial mesh, the standard lowest order finite element spaces provide a complex $X^{\bullet}$ centered on Raviart-Thomas divergence conforming vector fields. It can be seen as a realization of the simplicial cochain complex. We construct a new complex $Y^{\bullet}$ of finite element spaces on the barycentric refinement of the mesh which can be seen as a realization of the simplicial chain complex on the original (unrefined) mesh, such that the $\mathrm{L}^{2}$ duality is non-degenerate on $Y^{i} \times X^{2-i}$ for each $i \in\{0,1,2\}$. In particular $Y^{1}$ is a space of curl-conforming vector fields which is $\mathrm{L}^{2}$ dual to Raviart-Thomas div-conforming elements. When interpreted in terms of differential forms, these two complexes provide a finite-dimensional analogue of Hodge duality.
\end{abstract}

\section{INTRODUCTION}

Given a bounded domain in $\mathbb{R}^{3}$, many electromagnetic scattering problems can be reduced to integral equations on its surface $\Gamma$, whose unknowns are tangent vector fields. In the boundary element method these are solved approximately by introducing finite-element spaces of vector fields on $\Gamma$. In particular, the most successful space for solving the so-called electric field integral equation consists of surface Raviart-Thomas vector fields constructed on a triangulation of $\Gamma$ (see Bendali (4). We denote by $X^{1}$ this space of divergence conforming vector fields. For some problems, in particular for preconditioning the electric field integral equation and for some formulations of impedance boundary conditions, it would be useful to have a space $Y^{1}$ of curl conforming vector fields on $\Gamma$ which is $\mathrm{L}^{2}$ dual to $X^{1}$. In this paper we construct such a space, as a certain subspace of standard finite elements of the barycentric refinement of the original mesh. In fact we insert $X^{1}$ into a complex $X^{\bullet}$ and construct a complex $Y^{\bullet}$ such that the $\mathrm{L}^{2}$ duality on $Y^{i} \times X^{2-i}$ is non-degenerate for each $i \in\{0,1,2\}$.

We choose the orientation on $\Gamma$ induced by the outward pointing normal on $\Gamma$ (with respect to the interior, bounded domain), which we denote by $n$. It should be noticed that putting $Y^{1}=X^{1} \times n$ does not provide an adequate space, as remarked in [14.

The interpretation of the finite-element spaces of Raviart-Thomas type as spaces of differential forms (called Whitney forms) having the algebraic properties of the simplicial cochain complex of the mesh, was put forward by Bossavit 5 . From this

Received by the editor July 6, 2005 and, in revised form, July 25, 2006.

2000 Mathematics Subject Classification. Primary 65N30, 65N38.

(C)2007 American Mathematical Society

Reverts to public domain 28 years from publication 
point of view the spaces of scalar and vector fields we introduce are new (except perhaps $Y^{2}$ which is a variant of the space of piecewise constant fields on the Voronoi dual of the mesh) spaces of differential forms, but they have the algebraic properties of the simplicial chain complex of the mesh. However, as already indicated these spaces are constructed on a refinement of that mesh. Various differential complexes have been increasingly used to interpret and guide developments in numerical analysis, and we refer to Arnold 3] for an exposition of this topic. This paper can be seen as belonging to this trend. In this direction it provides an answer, in two dimensions, to the problem of constructing a finite-element analogue of Hodge duality ; indeed when the spaces $X^{i}$ and $Y^{i}$ are interpreted as spaces of differential forms, the duality on $Y^{i} \times X^{2-i}$ we show to be non-degenerate is $(v, u) \mapsto \int v \wedge u$.

In fact all the results of this paper are valid in the slightly more general setting of an arbitrary oriented piecewise linear two-dimensional manifold without boundary, but embedding it in $\mathbb{R}^{3}$ makes the presentation less technical and corresponds to the above applications. For the same reason we have chosen to present the results in terms of vector fields rather that differential forms. For completeness, at the end of the paper, we will come back to the general setting and also give the translation of our results into the language of differential forms, defining in particular a discrete analogue of the Hodge star operator.

The paper is organized as follows: In $₫ 2$ we define the new spaces $Y_{h}^{i}$ and show some algebraic properties pertaining to the complex they form. In 33 we prove approximation properties, estimates on discrete Hodge-Helmholtz decompositions in $Y_{h}^{1}$ and inf-sup conditions for the $\mathrm{L}^{2}$ dualities on $Y_{h}^{i} \times X_{h}^{2-i}$. In $\S 4$ we translate some of the results of this paper into the language of differential forms, provide an application to the preconditioning of the Electric Field Integral Equation and provide an extension of the construction to open surfaces. Some of these results were announced without proof in Buffa-Christiansen [10].

\section{Definition And Algebraic properties}

We consider a polyhedron in $\mathbb{R}^{3}$ whose boundary is locally the graph of a Lipschitz function, and denote this boundary by $\Gamma$ (the polyhedron itself will not be considered explicitly). Thus $\Gamma$ is a two-dimensional surface embedded in $\mathbb{R}^{3}$ and we equip it with a family of meshes $\left(\mathcal{T}_{h}\right)$ which is conforming and regular in the sense defined by Ciarlet in [17. For each $h$ the mesh $\mathcal{T}_{h}$ is simplicial, and for each integer $i$ we denote by $\mathcal{T}_{h}^{i}$ the subset of $\mathcal{T}_{h}$ consisting of $i$-dimensional simplexes (i.e. elements of cardinality $i+1$ ). The elements of $\mathcal{T}_{h}^{0}$ are the nodes or vertexes, the elements of $\mathcal{T}_{h}^{1}$ are the edges and those of $\mathcal{T}_{h}^{2}$ are the triangles of $\mathcal{T}_{h}$. For $s \in \mathcal{T}_{h}$ we denote by $|s|$ the convex envelope of $s$ in $\mathbb{R}^{3}$. When we need to distinguish a simplex $s$ from $|s|$, the latter will be called the geometric realization of $s$. We suppose that edges of the polyhedral surface $\Gamma$ do not cut any triangle of the mesh: the edges of $\Gamma$ are indeed union of (geometric realizations of) edges $e \in \mathcal{T}_{h}^{1}$.

The triangles of $\mathcal{T}_{h}$ are oriented by the outward pointing unit normal $n$ on $\Gamma$, and for each edge an orientation is chosen. The oriented unit-norm tangent vector along an edge $e$ is denoted $\tau_{e}$.

The barycentric refinement of $\mathcal{T}_{h}$ is defined by dividing each triangle $s \in \mathcal{T}_{h}^{2}$ into six triangles and drawing the six edges joining the barycenter of $s$ with the vertexes of $s$ as well as the midpoints of its edges. The barycentric refinement of $\mathcal{T}_{h}$ 
is denoted $\mathcal{T}_{h}^{\prime}$. In the Figures 1, 2 and 3 the edges of $\mathcal{T}_{h}$ are drawn in bold, whereas non-bold segments are edges of $\mathcal{T}_{h}^{\prime}$ (all bold segments are also edges of $\mathcal{T}_{h}^{\prime}$ ).

On $\mathcal{T}_{h}$ we consider the lowest order finite-element complex $\left(X_{h}^{0}, X_{h}^{1}, X_{h}^{2}\right)$ based on Raviart-Thomas divergence conforming vector fields $\mathrm{RT}_{0}$. It is defined by:

$$
\begin{aligned}
& X_{h}^{0}=\left\{u \in \mathrm{H}^{1}(\Gamma) \quad:\left.\forall t \in \mathcal{T}_{h}^{2} \quad u\right|_{t} \in \mathcal{P}_{1}\right\}, \\
& X_{h}^{1}=\left\{u \in \mathrm{H}_{\text {div }}(\Gamma):\left.\forall t \in \mathcal{T}_{h}^{2} \quad u\right|_{t} \in \mathrm{RT}_{0}\right\}, \\
& X_{h}^{2}=\left\{u \in \mathrm{L}^{2}(\Gamma) \quad:\left.\forall t \in \mathcal{T}_{h}^{2} \quad u\right|_{t} \in \mathcal{P}_{0}\right\} .
\end{aligned}
$$

For generalities about mixed finite elements and Raviart-Thomas vector fields in particular, we refer to Brezzi-Fortin [7. Such finite element spaces on surfaces were considered in the context of integral equations for electromagnetism in Bendali [4. For definitions of the functional spaces in the case of non-smooth surfaces we refer to Buffa-Ciarlet [11. Elements of $X_{h}^{0}$ are continuous and elements of $X_{h}^{1}$ have continuous normal component (flux) across edges. Thus, in particular, normal fluxes are continuous across the edges of the surface $\Gamma$. These spaces satisfy $\operatorname{curl} X_{h}^{0} \subset X_{h}^{1}$ and $\operatorname{div} X_{h}^{1} \subset X_{h}^{2}$, so that the spaces do indeed form a complex:

$$
X_{h}^{0} \stackrel{\text { curl }}{\longrightarrow} X_{h}^{1} \stackrel{\operatorname{div}}{\longrightarrow} X_{h}^{2}
$$

We denote by $\lambda^{i}=\left(\lambda_{s}^{i}\right)$ indexed by $s \in \mathcal{T}_{h}^{i}$ the standard basis of $X_{h}^{i}$. For each $i$, the usual family of degrees on freedom relative to $X_{h}^{i}$ will be denoted $l^{i}=\left(l_{s}^{i}\right)$ indexed by $s \in \mathcal{T}_{h}^{i}$. Then $l_{v}^{0}$ is evaluation at the vertex $v, l_{e}^{1}$ is integration of the normal component along the edge $e$ in some orientation, and $l_{t}^{2}$ is integration on the triangle $t$. In a sense, for each $i$ and each $s, l_{s}^{i}$ can be represented as integration on the simplex $s$. The basis $\lambda^{i}$ of $X_{h}^{i}$ is characterized by the property that $l_{s}^{i}\left(\lambda_{t}^{i}\right)=\delta_{s t}$.

On $\mathcal{T}_{h}^{\prime}$ we consider the slightly different finite-element complex $\left(\widetilde{X}_{h}^{0}, \widetilde{X}_{h}^{1}, \widetilde{X}_{h}^{2}\right)$ defined by:

$$
\begin{aligned}
& \widetilde{X}_{h}^{0}=\left\{u \in \mathrm{H}^{1}(\Gamma) \quad:\left.\forall t \in \mathcal{T}_{h}^{\prime 2} \quad u\right|_{t} \in \mathcal{P}_{1}\right\}, \\
& \widetilde{X}_{h}^{1}=\left\{u \in \mathrm{H}_{\text {curl }}(\Gamma):\left.\forall t \in \mathcal{T}_{h}^{\prime 2} \quad u\right|_{t} \in \mathrm{RT}_{0} \times n\right\}, \\
& \widetilde{X}_{h}^{2}=\left\{u \in \mathrm{L}^{2}(\Gamma) \quad:\left.\forall t \in \mathcal{T}_{h}^{2} \quad u\right|_{t} \in \mathcal{P}_{0}\right\} .
\end{aligned}
$$

The only difference from the spaces corresponding to $X_{h}^{i}$ on the refined mesh $\mathcal{T}_{h}^{\prime}$ is that we rotate the middle one by the operation $u \rightarrow u \times n$. These spaces satisfy $\operatorname{grad} \widetilde{X}_{h}^{0} \subset \widetilde{X}_{h}^{1}$ and $\operatorname{curl} \widetilde{X}_{h}^{1} \subset \widetilde{X}_{h}^{2}$ so that we have the complex:

$$
\widetilde{X}_{h}^{0} \stackrel{\operatorname{grad}}{\longrightarrow} \widetilde{X}_{h}^{1} \stackrel{\text { curl }}{\longrightarrow} \widetilde{X}_{h}^{2} \text {. }
$$

Bases are constructed for the spaces $\widetilde{X}_{h}^{i}$ associated with $\mathcal{T}_{h}^{\prime}$ as for the spaces associated with $\mathcal{T}_{h}$, and denoted $\left(\widetilde{\lambda}_{s}^{i}: s \in \mathcal{T}_{h}^{\prime i}\right)$ (the corresponding degrees of freedom will not be needed).

The aim of this paper is to construct subspaces $Y_{h}^{i} \subset \widetilde{X}_{h}^{i}$ such that on the one hand $Y_{h}^{i}$ is $\mathrm{L}^{2}$-dual to $X_{h}^{2-i}$ (in the sense of satisfying a Babuska-Brezzi Inf-Sup condition uniformly in $h$, in appropriate norms), and on the other hand they should form a complex:

$$
\mathrm{Y}_{h}^{0} \stackrel{\operatorname{grad}}{\longrightarrow} \mathrm{Y}_{h}^{1} \stackrel{\text { curl }}{\longrightarrow} \mathrm{Y}_{h}^{2} .
$$

We define these spaces by the construction of a spanning family and then check that it is a basis and that our above goals are fulfilled. For each $i \in\{0,1,2\}$ and 
each simplex $s \in \mathcal{T}_{h}^{2-i}$, let $\mu_{s}^{i} \in \widetilde{X}_{h}^{i}$ be the field attached to $s$ constructed as a linear combination of the basis functions $\widetilde{\lambda}_{t}^{i}$ of $\widetilde{X}_{h}^{i}$, with the following coefficients:

- For $i=0$ the coefficients are shown in Figure 1 $s$ is the triangle of $\mathcal{T}_{h}$ whose barycenter carries the coefficient 1 . Thus $\mu_{s}^{0}$ is the element of $\widetilde{X}_{h}^{0}$ with non-zero values at the vertices shown in that figure.

- For $i=1$ the coefficients are shown in Figure $2 s$ is the central edge, and we have oriented the edges as pointing away from it. Thus $\mu_{s}^{1}$ is an element of $\widetilde{X}_{h}^{1}$ such that the integrals of the tangent component on edges is the coefficient shown in the figure. The coefficient of each edge should be multiplied by the one indicated at its origin, e.g., to the left we have coefficients ranging from $5 / 12$ to $-5 / 12$ when ordered counter-clockwise. Edges not carrying a value are given the coefficient 0 ; this is in particular the case for the two small edges composing the central edge $s$.

- For $i=2$ the coefficients are shown in Figure $3 s$ is the central vertex. All 12 triangles of $\mathcal{T}_{h}^{\prime}$ in the shaded region should carry the same weight $1 / 12$. Thus $\mu_{s}^{2}$ is the element in $\widetilde{X}_{h}^{2}$ whose integral is $1 / 12$ on each shaded triangle, and 0 elsewhere.

In each figure the shaded region is the support of the corresponding field.

We define $Y_{h}^{i}$ by:

$$
Y_{h}^{i}=\operatorname{span}\left\{\mu_{s}^{i}: s \in \mathcal{T}_{h}^{2-i}\right\} .
$$

For each integer $i \in\{0,1,2\}$, we now construct families of linear forms on fields (scalar or vector according to the case) whose restrictions to $\mathrm{Y}_{h}^{i}$ are linearly independent. These linear forms are the degrees of freedom (dof).

To this aim we fix some notations. For each triangle $t \in \mathcal{T}_{h}^{2}$, let $t^{\prime}$ denote its barycenter. For each edge $e \in \mathcal{T}_{h}^{1}$, let $e^{\prime}$ be union of (the geometric realizations of) the two edges of $\mathcal{T}_{h}^{\prime}$ joining the barycenter of $e$ to the barycenters of the two neighboring triangles. The oriented tangent vector along $e^{\prime}$ is denoted $\tau_{e^{\prime}}$, orientation being chosen such that $\tau_{e^{\prime}} \cdot \tau_{e} \times n<0$. For each vertex $v \in \mathcal{T}_{h}^{0}$, denote by $v^{\prime}$ the union of (the geometric realizations of) the triangles of $\mathcal{T}_{h}^{\prime}$ containing $v$.

We now define three families of degrees of freedom:

$$
\begin{aligned}
& \mathrm{M}_{h}^{0}=\left(m_{t}^{0}: u \mapsto u\left(t^{\prime}\right) \quad: t \in \mathcal{T}_{h}^{2}\right), \\
& \mathrm{M}_{h}^{1}=\left(m_{e}^{1}: u \mapsto \int_{e^{\prime}} u \cdot \tau_{e^{\prime}}: e \in \mathcal{T}_{h}^{1}\right), \\
& \mathrm{M}_{h}^{2}=\left(m_{v}^{2}: u \mapsto \int_{v^{\prime}} u \quad: v \in \mathcal{T}_{h}^{0}\right) .
\end{aligned}
$$

We remark that the first family of linear forms $\mathrm{M}_{h}^{0}$ can also be written as integrals (with respect to the trivial measure on points). In this sense the three preceding definitions may be written:

$$
\mathrm{M}_{h}^{i}=\left(m_{s}^{i}: u \mapsto \int_{s^{\prime}} u: s \in \mathcal{T}_{h}^{2-i}\right),
$$

where we integrate on certain dual geometric objects $s^{\prime}$ relative to $\mathcal{T}_{h}^{\prime}$ and attached to simplexes $s \in \mathcal{T}_{h}$, defined above.

Proposition 2.1. For each $i \in\{0,1,2\}$ and each $i$-dimensional simplexes $s, t \in \mathcal{T}_{h}^{i}$ we have:

$$
m_{s}^{i}\left(\mu_{t}^{i}\right)=\delta_{s t} .
$$




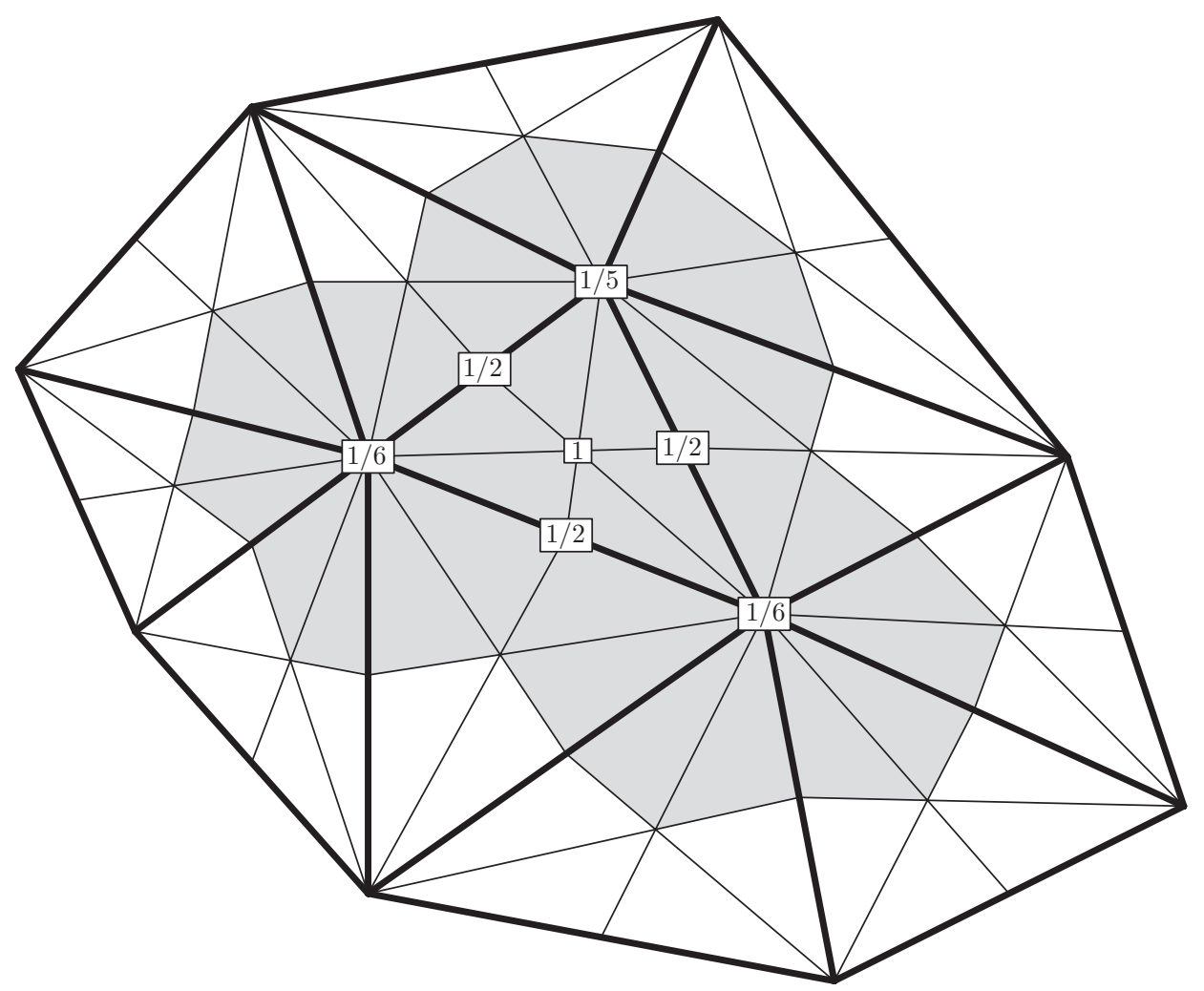

Figure 1. A basis element for $Y_{h}^{0}$ expressed in the basis of $\widetilde{X}_{h}^{0}$

In particular, for each $i$ the family $\mu^{i}=\left(\mu_{s}^{i}\right)$ indexed by $s \in \mathcal{T}_{h}^{2-i}$ is a basis for $Y_{h}^{i}$, and an element $u \in \mathrm{Y}_{h}^{i}, i=0,1,2$, is uniquely determined the values $m_{s}^{i}(u)$ for $s \in \mathcal{T}_{h}^{2-i}$.

Proof. This is a matter of straightforward checking.

We also remark that:

Proposition 2.2. The family of functions $\left(\mu_{s}^{0}: s \in \mathcal{T}_{h}^{2}\right)$ is a partition of unity.

Proof. It is enough to remark that for each $s \in \mathcal{T}_{h}^{2}$ the nonzero values of $\mu_{s}^{0}$ at the vertexes $v$ of the barycentric refinement $\mathcal{T}_{h}^{\prime}$ are the inverses of the number of triangles $t \in \mathcal{T}_{h}^{2}$ such that $v \in|t|$. Therefore the sum of the functions $\mu_{s}^{0}$ evaluated at any such vertex $v$ is 1 .

Proposition 2.3. We have grad $\mathrm{Y}_{h}^{0} \subset \mathrm{Y}_{h}^{1}$ and $\operatorname{curl} Y_{h}^{1} \subset \mathrm{Y}_{h}^{2}$. Moreover the matrix of $\operatorname{grad}: \mathrm{Y}_{h}^{0} \rightarrow \mathrm{Y}_{h}^{1}$ in the basis $\mu^{0} \rightarrow \mu^{1}$ is minus the transpose of the matrix of div : $X_{h}^{1} \rightarrow X_{h}^{2}$ in the standard basis, and similarly the matrix of curl $: Y_{h}^{1} \rightarrow \mathrm{Y}_{h}^{2}$ in the basis $\mu^{1} \rightarrow \mu^{2}$ is the transpose of the matrix of curl : $X_{h}^{0} \rightarrow X_{h}^{1}$ in the standard basis. 


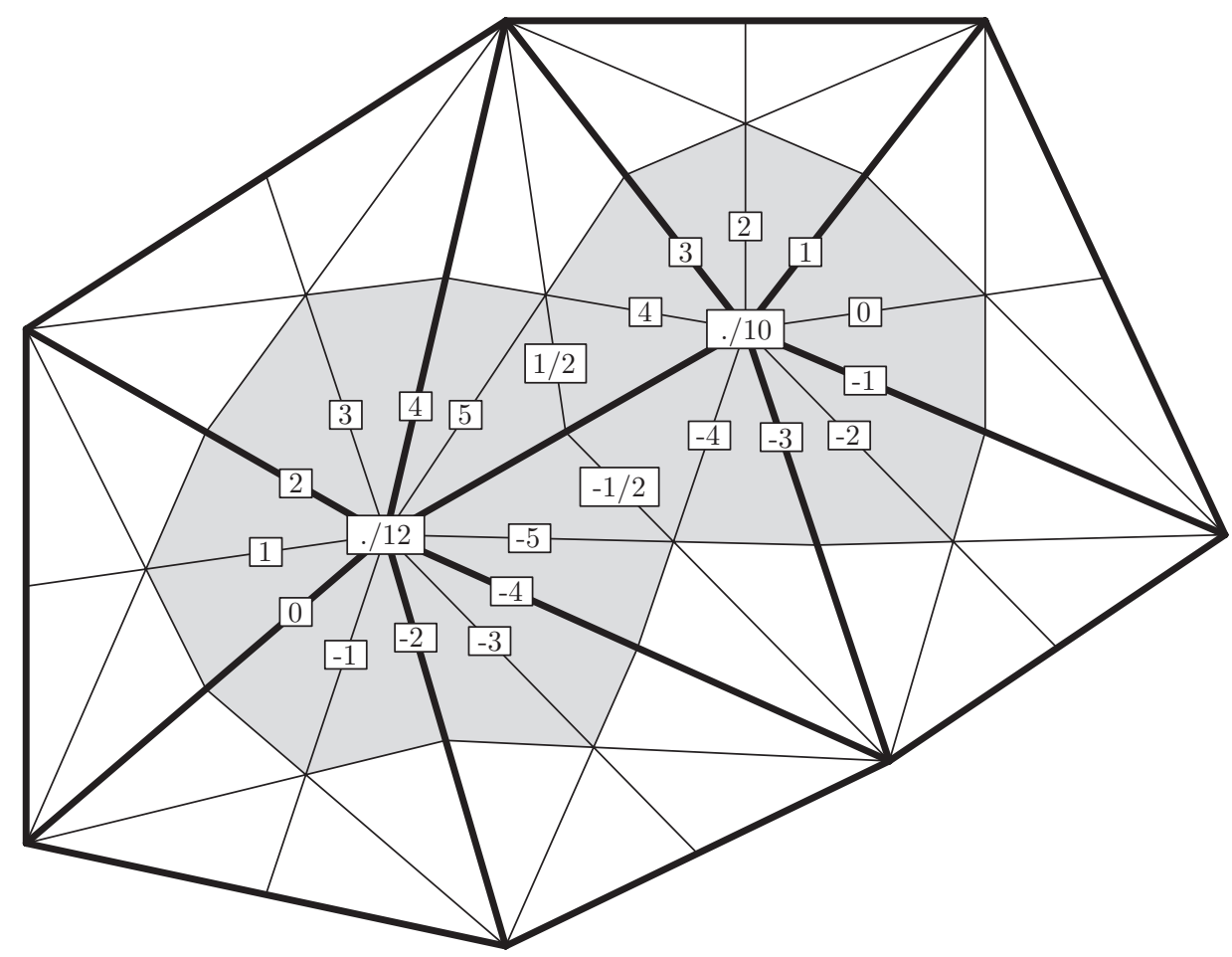

Figure 2. A basis element for $Y_{h}^{1}$ expressed in the basis of $\widetilde{X}_{h}^{1}$.

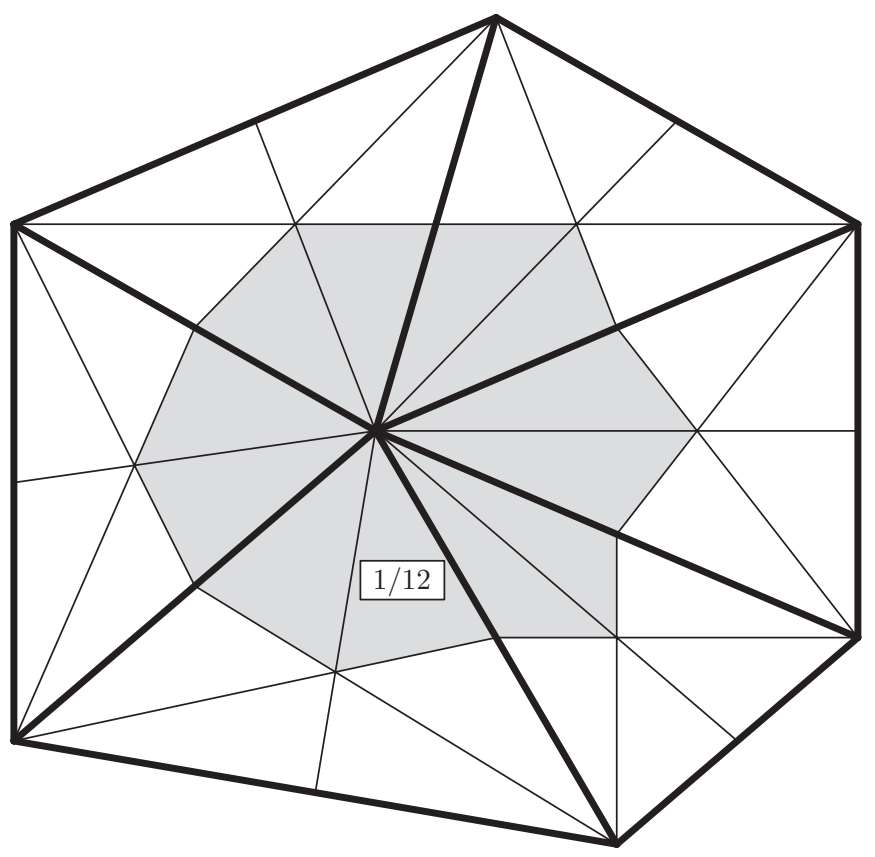

Figure 3. A basis element for $Y_{h}^{2}$ expressed in the basis of $\widetilde{X}_{h}^{2}$. 
Proof. Concerning the grad operator, one checks that for each triangle $t \in \mathcal{T}_{h}$, $\operatorname{grad} \mu_{t}^{0}$ is a linear combination of the three vector-fields $\mu_{e}^{1}$ where $e$ is an edge of $t$. The coefficients are 1 or -1 according to orientations of the edges. Checking this is a matter of elementary but tedious computations using only the definitions of basis functions. The matrix thus formed is known as an incidence matrix, and its transpose is also known to be the matrix of $-\operatorname{div}: X_{h}^{1} \rightarrow X_{h}^{2}$ in the standard basis.

The case of the curl operator is similar.

For each $i \in\{0,1,2\}$ we denote by $\mathrm{I}_{h}^{i}$ the interpolation operator associated with the d.o.f. $\mathrm{M}_{h}^{i}$. Explicitly $\mathrm{I}_{h}^{i}$ associates with a field $u$ (scalar or vector according to i) the element $u_{h}$ of $Y_{h}^{i}$ such that:

$$
\forall s \in \mathcal{T}_{h}^{2-i} \quad m_{s}^{i}\left(u_{h}\right)=m_{s}^{i}(u) .
$$

Let $\Omega^{0} \subset \mathrm{H}^{1}(\Gamma), \Omega^{1} \subset \mathrm{H}_{\text {curl }}(\Gamma)$ and $\Omega^{2} \subset \mathrm{L}^{2}(\Gamma)$ be the subspaces consisting of piecewise smooth fields. We then have:

Proposition 2.4. The interpolators satisfy the following commuting diagram:

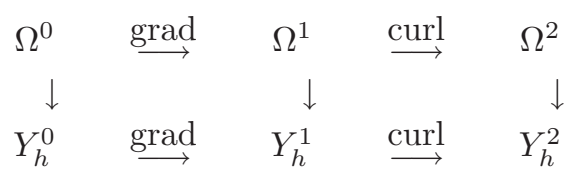

Proof. This follows from an application of Stokes' theorem on the geometric elements $s^{\prime}$ we associated with the simplexes $s \in \mathcal{T}_{h}$ in order to define the degrees of freedom.

Moreover:

Proposition 2.5. In the following complex, the cohomology groups have the "right" dimension:

$$
0 \longrightarrow \mathrm{Y}_{h}^{0} \stackrel{\operatorname{grad}}{\longrightarrow} \mathrm{Y}_{h}^{1} \stackrel{\text { curl }}{\longrightarrow} \mathrm{Y}_{h}^{2} \longrightarrow 0
$$

Specifically, if $\Gamma$ is connected, for the first cohomology group an element of $Y_{h}^{0}$ has gradient 0 iff it is constant, whereas for the last cohomology group an element of $Y_{h}^{2}$ is the curl of an element of $Y_{h}^{1}$ iff it has 0 integral.

Proof. This can be deduced from the fact that the complex formed by the spaces $\left(X_{h}^{i}\right)$ is known to have cohomology groups of the right dimension (the dimension of the DeRham cohomology groups) and that the matrices of the operators linking the spaces $Y_{h}^{i}$ are transposes of the matrices of the operators linking the spaces $X_{h}^{i}$ (in the choices of basis we have used), as we have already remarked. 1

Remark 2.1. The appearance of the incidence matrices in the proof of Proposition 2.3 can be interpreted as the fact that we have a commuting diagram:

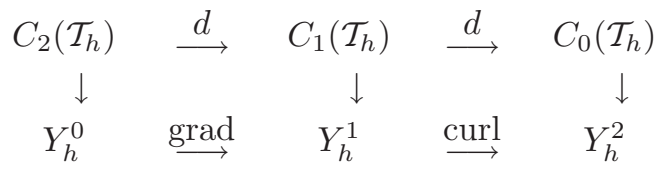

\footnotetext{
${ }^{1}$ Given two matrices $A$ and $B$ such that $A B=0$ we have $B^{\mathrm{T}} A^{\mathrm{T}}=0$ and $\operatorname{dim}((\operatorname{ker} A) /(\operatorname{ran} B))=\operatorname{dim}((\operatorname{ker} A) /(\operatorname{ran} B))^{\star}=\operatorname{dim}(\operatorname{ran} B)^{\circ} /(\operatorname{ker} A)^{\circ}=\operatorname{dim}\left(\operatorname{ker} B^{T}\right) /\left(\operatorname{ran} A^{T}\right)$.
} 
where the complex on the upper line is the simplicial chain complex (see GelfandManin 20] p. 23) and the vertical arrows are the basis interpretation isomorphisms:

$$
\left\{\begin{aligned}
C_{i}\left(\mathcal{T}_{h}\right) \approx \mathbb{R}^{\mathcal{T}_{h}^{i}} & \rightarrow Y_{h}^{2-i}, \\
\left(c: \mathcal{T}_{h}^{i} \rightarrow \mathbb{R}\right) & \mapsto \sum_{s \in \mathcal{T}_{h}^{i}} c(s) \mu_{s}^{i} .
\end{aligned}\right.
$$

As remarked by Bossavit $[5$ there is a similar diagram linking the simplicial cochain complex to the standard spaces $X_{h}^{i}$.

\section{Metric properties}

This section is devoted to the metric properties of the complex $Y_{h}^{\bullet}=\left(\mathrm{Y}_{h}^{0}, \mathrm{Y}_{h}^{1}, \mathrm{Y}_{h}^{2}\right)$. More precisely we investigate its approximation properties, stability (with respect to the mesh size) of the Helmholtz-Hodge decompositions related to Proposition 2.5. and of the dualities on $Y_{h}^{i} \times X_{h}^{i-2}, i=0,1,2$, in natural norms. To this aim, we introduce a few notations and spaces. In what follows $\mathrm{H}^{s}(\Gamma), s \in\left[-1, \frac{3}{2}\right)$, denotes the standard Sobolev space of $\Gamma$ of Sobolev regularity $s$ endowed with the norm $\|\cdot\|_{s}$ and seminorm $|\cdot|_{s}$ (the domain will be specified when different from $\Gamma$, e.g., $\left.|\cdot|_{s, v^{\prime}}, v \in \mathcal{T}_{h}^{0}\right)$. Moreover, for $s \in\left(-1, \frac{1}{2}\right)$ we set:

$$
\begin{aligned}
\mathrm{H}_{\text {curl }}^{s}(\Gamma) & :=\left\{u \in \mathrm{H}^{s}(\Gamma): \operatorname{curl} u \in \mathrm{H}^{s}(\Gamma)\right\}, \\
\mathrm{H}_{\text {div }}^{s}(\Gamma) & :=\left\{u \in \mathrm{H}^{s}(\Gamma): \operatorname{div} u \in \mathrm{H}^{s}(\Gamma)\right\},
\end{aligned}
$$

These spaces are endowed with their graph norms $\|\cdot\|_{s, \text { curl }}$ and $\|\cdot\|_{s, \text { div }}$, respectively. For details about these spaces we refer the reader to Buffa [8]. Moreover, all along this section, the symbol $\cdot \lesssim$ will stand for $\cdot \leq C \cdot$, where $C$ is a generic constant depending only on $\Gamma$ (not on $h$ ).

3.1. Error estimates. For each integer $N$ we denote by $\widehat{C}_{N}$ a regular polygon with $N$ sides (edges) of length 1 and having the barycentre at the origin of the chosen Cartesian system.

For each node $v \in \mathcal{T}_{h}^{0}$ we denote by $N_{v}$ the number of triangles in $\mathcal{T}_{h}^{2}$ containing $v$ and pick a bi-Lipschitz transformation $F_{v}: v^{\prime} \rightarrow \widehat{C}_{N_{v}}$ from the cell $v^{\prime}$ dual to $v$ (thus $v^{\prime}$ is the support of $\mu_{v}^{2}$ ) which is piecewise affine with respect to $\mathcal{T}_{h}^{\prime}$ such that:

(1) the barycentre of the triangles in $\mathcal{T}_{h}$ meeting $v^{\prime}$ are sent to vertices of $\widehat{C}_{N_{v}}$;

(2) the barycentre of the edges of $\mathcal{T}_{h}$ meeting $v^{\prime}$ are sent to the barycentre of the sides of $\widehat{C}_{N_{v}}$;

(3) $v$ is sent to the barycentre of $\widehat{C}_{N_{v}}$.

Note that, since the mesh $\mathcal{T}_{h}$ is supposed regular, there is a constant $\mathcal{N}$ independent of the mesh size, such $N_{v} \leq \mathcal{N}$ for all $v \in \mathcal{T}_{h}^{0}$. For a given $N$, we denote by $\widehat{x}_{\ell}, \ell=1, \ldots, N$, the vertices of $\widehat{C}_{N}$ (numbered in a counterclockwise sense), and by $\widehat{x}_{\ell+1 / 2}, \ell=1, \ldots, N$, the mid-points of the segment $\left(\widehat{x}_{\ell}, \widehat{x}_{\ell+1}\right)$, where the indexes are taken modulo $N$. Moreover, $\tau_{0, \widehat{x}_{\ell}}$ denotes the tangent vector along the oriented segment $\left(0, \widehat{x}_{\ell}\right)$ and $\tau_{\widehat{x}_{\ell}, \widehat{x}_{\ell+1}}$ the tangent vector along the oriented segment $\left(\widehat{x}_{\ell}, \widehat{x}_{\ell+1}\right)$.

We denote by $\mathfrak{F}_{v}: \widehat{C}_{N} \rightarrow v^{\prime}$ the inverse of $F_{v}$, which is again a Lipschitz piecewise affine mapping. More precisely, for each $t \in \mathcal{T}_{h}^{\prime 2} \cap v^{\prime},\left.F_{v}\right|_{t}$ is affine, invertible and we denote by $\mathfrak{F}_{t}, \mathfrak{F}_{t}(\widehat{x})=\mathfrak{B}_{t} \widehat{x}+\mathfrak{c}_{t}$, its inverse. Standard scaling arguments say that:

$$
\left\|\mathfrak{B}_{t}\right\| \lesssim h,\left|\operatorname{det} \mathfrak{B}_{t}\right| \lesssim h^{2}, \quad\left\|\mathfrak{B}_{t}^{-1}\right\| \lesssim h^{-1},\left|\operatorname{det} \mathfrak{B}_{t}^{-1}\right| \lesssim h^{-2}
$$


For each $u^{0} \in \mathrm{H}^{1+s}(\Gamma), u^{1} \in \mathrm{H}_{\text {curl }}^{s}(\Gamma), u^{2} \in \mathrm{H}^{s}(\Gamma)$ we define the pull-back mappings as follows:

$$
\widehat{u}^{0}=u^{0} \circ \mathfrak{F}_{v}, \widehat{u}^{1}=D \mathfrak{F}_{v}^{T}\left(u^{1} \circ \mathfrak{F}_{v}\right), \widehat{u}^{2}=\operatorname{det} D \mathfrak{F}_{v} u^{2} \circ \mathfrak{F}_{v} .
$$

The pull-back operators involve the Lipschitz piecewise affine mapping $\mathfrak{F}_{v}$. Thus, they preserve only Sobolev regularity for "small" exponents. More precisely, $\widehat{u}^{0} \in$ $\mathrm{H}^{1+s}\left(\widehat{C}_{N}\right), \widehat{u}^{1} \in \mathrm{H}_{\text {curl }}^{s}\left(\widehat{C}_{N}\right), \widehat{u}^{2} \in \mathrm{H}^{s}\left(\widehat{C}_{N}\right)$ only for $s<\frac{1}{2}$. Finally, by scaling argument, we obtain the following proposition:

Proposition 3.1. Let $u^{0} \in \mathrm{H}^{1+s}(\Gamma), u^{1} \in \mathrm{H}_{\text {curl }}^{s}(\Gamma)$, and fix $v \in \mathcal{T}_{h}^{0}$. We denote by $\widehat{u}^{i}$ the pull-backs of $u^{i}$ obtained by means of the mappings (17). The following scaling estimates hold for each $0 \leq s<\frac{1}{2}$ :

$$
\begin{aligned}
\left|\widehat{u}^{0}\right|_{1+s, \widehat{C}_{N}} & \leq C(s, N) h^{s}\left|u^{0}\right|_{1+s, v^{\prime}} \\
\left|\widehat{u}^{1}\right|_{s, \widehat{C}_{N}} & \leq C(s, N) h^{s}\left|u^{1}\right|_{s, v^{\prime}}, \\
\left|\operatorname{curl} \widehat{u}^{1}\right|_{s, \widehat{C}_{N}} & \leq C(s, N) h^{1+s}\left|\operatorname{curl} u^{1}\right|_{s, v^{\prime}}
\end{aligned}
$$

where the constant $C(s, N)$ does not depend upon $u^{i}$.

Proof. We concentrate on (18b) and (18c). The scaling estimate (18a) can be obtained by the same arguments. Moreover, for $s=0$, the proof is easy and then omitted. We fix $s: 0<s<\frac{1}{2}, v \in \mathcal{T}_{h}^{0}$, and a function $u^{1} \in \mathrm{H}_{\text {curl }}^{s}(\Gamma)$. For each $t \in \mathcal{T}_{h}^{\prime 2} \cap v^{\prime}$ we denote by $\widehat{t}$ the triangle in $\widehat{C}_{N}$ such that $t=\mathfrak{B}_{t} \widehat{t}+\mathfrak{c}_{t}$.

(i) Proof of $18 \mathrm{~b}$.

$$
\begin{aligned}
\left|\widehat{u}^{1}\right|_{s, \widehat{C}_{N}}^{2} & \lesssim \sum_{\widehat{t} \in \widehat{C}_{N}}\left|\widehat{u}^{1}\right|_{s, \widehat{t}}^{2} \quad \text { since } s<\frac{1}{2} \\
& \lesssim \sum_{t \in v^{\prime}}\left|\operatorname{det} \mathfrak{B}_{t}\right|^{-2} \int_{t} \int_{t} \frac{\left|\mathfrak{B}_{t}^{T}\left(u^{1}(x)-u^{1}(y)\right)\right|^{2}}{\left|\mathfrak{B}_{t}^{-1}(x-y)\right|^{2+2 s}} \\
& \lesssim \sum_{t \in v^{\prime}}\left|\operatorname{det} \mathfrak{B}_{t}\right|^{-2}\left\|\mathfrak{B}_{t}\right\|^{4+2 s} \int_{t} \int_{t} \frac{\left|u^{1}(x)-u^{1}(y)\right|^{2}}{|x-y|^{2+2 s}} \\
& \lesssim \sum_{t \in v^{\prime}} h^{2 s}\left|u^{1}\right|_{s, t}^{2} \leq C h^{2 s}\left|u^{1}\right|_{s, v^{\prime}}^{2} .
\end{aligned}
$$

(ii) Proof of (18c). This proof is inspired by the corresponding one for standard edge elements; see Alonso-Valli [1]. Using (17) it is easy to deduce that curl $\widehat{u}^{1}=$ $\left(\operatorname{det} D \mathfrak{F}_{v}\right)\left(\operatorname{curl} u^{1}\right) \circ \mathfrak{F}_{v}$. We then estimate:

$$
\begin{aligned}
\left|\operatorname{curl} \widehat{u}^{1}\right|_{s, \widehat{C}_{N}}^{2} & \lesssim \sum_{\hat{t} \in \widehat{C}_{N}}\left|\operatorname{curl} \widehat{u}^{1}\right|_{s, \widehat{t}}^{2} \quad \text { since } s<\frac{1}{2} \\
& \lesssim \sum_{t \in v^{\prime}}\left|\operatorname{det} \mathfrak{B}_{t}\right|^{-2} \int_{t} \int_{t} \frac{\left|\operatorname{det} \mathfrak{B}_{t}\left(\operatorname{curl} u^{1}(x)-\operatorname{curl} u^{1}(y)\right)\right|^{2}}{\left|\mathfrak{B}_{t}^{-1}(x-y)\right|^{2+2 s}} \\
& \lesssim \sum_{t \in v^{\prime}}\left|\operatorname{det} \mathfrak{B}_{t}\right|^{-2}|| \mathfrak{B}_{t} \|^{2+2 s}\left|\operatorname{det} \mathfrak{B}_{t}\right|^{2} \int_{t} \int_{t} \frac{\left|\operatorname{curl} u^{1}(x)-\operatorname{curl} u^{1}(y)\right|^{2}}{|x-y|^{2+2 s}} \\
& \lesssim \sum_{t \in v^{\prime}} h^{2+2 s}\left|\operatorname{curl} u^{1}\right|_{s, t}^{2} \leq C h^{2+2 s}\left|\operatorname{curl} u^{1}\right|_{s, v^{\prime}}^{2}
\end{aligned}
$$


Theorem 3.2. Let $0<s<\frac{1}{2}$. For any $u^{0} \in \mathrm{H}^{1+s}(\Gamma), u^{1} \in \mathrm{H}_{\text {curl }}^{s}(\Gamma), u^{2} \in \mathrm{H}^{s}(\Gamma)$, it holds that

$$
\begin{aligned}
\left\|u^{0}-\mathrm{I}_{h}^{0} u^{0}\right\|_{1}+h^{-1}\left\|u^{0}-\mathrm{I}_{h}^{0} u^{0}\right\|_{0} & \lesssim h^{s}\left\|u^{0}\right\|_{s+1}, \\
\left\|u^{1}-\mathrm{I}_{h}^{1} u^{1}\right\|_{0, \text { curl }} & \lesssim h^{s}\left\|u^{1}\right\|_{s, \text { curl }}, \\
\left\|u^{2}-\mathrm{I}_{h}^{2} u^{2}\right\|_{0} & \lesssim h^{s}\left\|u^{2}\right\|_{s} .
\end{aligned}
$$

Proof. First of all, given a $v \in \mathcal{T}_{h}^{0}$ and $u^{i} \in \mathrm{Y}_{h}^{i}(i=0,1,2)$, it is easy to see that $\left.u^{i}\right|_{v^{\prime}}$ is completely characterized by the values of $u^{i}$ on the boundary of $v^{\prime}$. More precisely, $\left.u^{0}\right|_{v^{\prime}}$ depends only on the nodal values at the barycentre falling on the boundary of $v^{\prime}, u^{1}$ on the moments $m_{s}^{1}$ on the segments on the boundary of $v^{\prime}$.

(i) Spaces on the reference cell. Fix a $v \in \mathcal{T}_{h}^{0}$, and let $\widehat{C}_{N}$ be the reference cell $\widehat{C}_{N}=F_{v}\left(v^{\prime}\right)$. We also map the basis functions $\mu^{i}$ with support meeting $v^{\prime}$ according to (17), and we number them according to the numbering given to the vertices of $\widehat{C}_{N}$. So, $\widehat{\mu}_{\ell}^{0}$ is the pull-back to $\widehat{C}_{N}$ of the basis function associated with the triangle $s$ such that $s^{\prime}=\mathfrak{F}_{v}\left(\widehat{x}_{\ell}\right), \widehat{\mu}_{\ell+1 / 2}^{1}$ is the pull-back to $\widehat{C}_{N}$ of the basis function associated with the segment $s$ such that $s^{\prime}=\mathfrak{F}_{v}\left(\left(\widehat{x}_{\ell}, \widehat{x}_{\ell+1}\right)\right)$ and, finally, $\widehat{\mu}^{2}$ is the pull-back of $\mu_{v^{\prime}}^{2}$.

The spaces on the reference cells $\widehat{C}_{N}$ are given by:

$$
\begin{aligned}
& \widehat{\mathrm{Y}}^{0}=\operatorname{Span}\left\{\widehat{\mu}_{\ell}^{0}\right\}, \\
& \widehat{\mathrm{Y}}^{1}=\operatorname{Span}\left\{\widehat{\mu}_{\ell+1 / 2}^{1}\right\}, \\
& \widehat{\mathrm{Y}}^{2}=\operatorname{Span}\left\{\widehat{\mu}^{2}\right\} .
\end{aligned}
$$

We remark that these spaces do not depend on the particular choice of $v \in \mathcal{T}_{h}^{0}$ which we used to define them.

Each function $\widehat{u}^{0} \in \widehat{\mathrm{Y}}^{0}$ verifies:

$$
\widehat{u}^{0}\left(\widehat{x}_{i, i+1}\right)=\frac{\widehat{u}^{0}\left(\widehat{x}_{i}\right)+\widehat{u}^{0}\left(\widehat{x}_{i+1}\right)}{2}, \widehat{u}^{0}(0)=\frac{1}{N} \sum_{i=1}^{n} \widehat{u}^{0}\left(\widehat{x}_{i}\right) .
$$

Each vector valued function $\widehat{u}^{1} \in \widehat{\mathrm{Y}}^{1}$ consists of an edge element, piecewise affine on each of the $2 N$ triangles (of the refined mesh) $\widehat{t}$ of $\widehat{C}_{N}$, such that its curl is constant on the cell $\widehat{C}_{N}$.

(i) Mappings. Fix $v \in \mathcal{T}_{h}^{0}$. We map functions and interpolation operators from $v^{\prime}$ to $\widehat{C}_{N}$ (for a suitable $N$ ) through the mappings (17). Thus:

$$
\widehat{\mathrm{I}}^{0}\left(\widehat{u}^{0}\right)=\mathrm{I}_{h}^{0} u^{0} \circ \mathfrak{F}_{v}, \widehat{\mathrm{I}}^{1} \widehat{u}^{1}=D \mathfrak{F}_{v}^{T}\left(\mathrm{I}_{h}^{1} \widehat{u}^{1} \circ \mathfrak{F}_{v}\right), \widehat{\mathrm{I}}^{2} \widehat{u}^{2}=\left(\operatorname{det} D \mathfrak{F}_{v}\right) \mathrm{I}_{h}^{2} u^{2} \circ \mathfrak{F}_{v} .
$$

By construction, we have that

$$
\widehat{\mathrm{I}}^{0}: \mathrm{H}^{1+s}\left(\widehat{C}_{N}\right) \rightarrow \widehat{\mathrm{Y}}^{0} \widehat{\mathrm{I}}^{1}: \mathrm{H}_{\text {curl }}^{s}\left(\widehat{C}_{N}\right) \rightarrow \widehat{\mathrm{Y}}^{1}, \widehat{\mathrm{I}}^{2}: \mathrm{H}^{s}\left(\widehat{C}_{N}\right) \rightarrow \widehat{\mathrm{Y}}^{2}
$$

are linear and continuous for each $s>0$.

Finally, $\widehat{\mathrm{Y}}^{\bullet}$ forms a complex (as in Proposition 2.5):

$$
0 \longrightarrow \widehat{\mathrm{Y}}^{0} \stackrel{\operatorname{grad}}{\longrightarrow} \widehat{\mathrm{Y}}^{1} \stackrel{\text { curl }}{\longrightarrow} \widehat{\mathrm{Y}}^{2} \longrightarrow 0 .
$$

(ii) Reproducing polynomial property. Fix $N$, and let $p^{0}$ be a polynomial of degree 1 defined on the reference cell $\widehat{C}_{N}$. Then $p^{0} \in \widehat{Y}^{0}$ thanks to (21). 
On the other hand, let $p^{1}$ be a Nédélec finite element on the cell $\widehat{C}_{N}$, i.e., $p^{1}(\widehat{x})=$ $\left(\begin{array}{c}a-b \widehat{x}_{2} \\ c+b \widehat{x}_{1}\end{array}\right)$. Then, $p^{1} \in \widehat{\mathrm{Y}}^{1}$. First, in the case $b=0$ : there exists a $p^{0} \in \widehat{\mathrm{Y}}^{0}$ such that $p^{1}=\operatorname{grad} p^{0}$. Indeed $p^{0}$ is a polynomial of degree 1 on the cell. Second, we treat the case $a=c=0$. We first remark that $\int_{0}^{\widehat{x}_{\ell}} p^{1} \cdot \tau_{0, \widehat{x}_{\ell}}=0$ for all $\ell$. Since $2 b=\operatorname{curl} p^{1}$ is constant on the cell, then a simple computation shows that $\int_{\left(\widehat{x}_{\ell}, \widehat{x}_{\ell+1}\right)} p^{1} \cdot \tau_{\widehat{x}_{\ell}, \widehat{x}_{\ell+1}}=N^{-1} 2 b\left|\widehat{C}_{N}\right|$. Thus $p^{1}=\sum_{\ell} N^{-1} 2 b\left|\widehat{C}_{N}\right| \widehat{\mu}_{\ell+1 / 2} \in \widehat{\mathrm{Y}}^{1}$.

(iii) Error estimate for $\mathrm{I}_{h}^{0}$. Let $u^{0} \in \mathrm{H}^{1+s}(\Gamma)\left(0<s<\frac{1}{2}\right), v \in \mathcal{T}_{h}^{0}$, and $\widehat{u}^{0}$ be the pull-back of $\left.u\right|_{v^{\prime}}$ on the reference cell $\widehat{C}_{N}$ for a suitable $N$. Note that, $\widehat{u} \in H^{1+s}\left(\widehat{C}_{N}\right)$ for $0 \leq s<\frac{1}{2}$. For each $t \in \mathcal{T}_{h}^{\prime 2} \cap v^{\prime}$, we have:

$$
\begin{aligned}
\left\|u^{0}-\mathrm{I}_{h}^{0} u^{0}\right\|_{0, t}^{2} & \lesssim\left|\operatorname{det} \mathfrak{B}_{t}\right|\left\|\widehat{u}^{0}-\widehat{\mathrm{I}}^{0} \widehat{u}^{0}\right\|_{0, \widehat{C}_{N}}^{2} \\
& \lesssim\left|\operatorname{det} \mathfrak{B}_{t}\right|\left|\widehat{u}^{0}\right|_{1+s, \widehat{C}_{N}}^{2} \lesssim h^{2(1+s)}\left|u^{0}\right|_{1+s, v^{\prime}}^{2}
\end{aligned}
$$

where we have used the reproducing polynomial property, Deny-Lions Lemma and (18a). The estimate for the $\mathrm{H}^{1}(\Gamma)$ norm can be obtained in a similar way.

(iv) Error estimate for $\mathrm{I}_{h}^{1}$ and $\mathrm{I}_{h}^{2}$. Let $u^{1} \in \mathrm{H}_{\text {curl }}^{s}(\Gamma)\left(0<s<\frac{1}{2}\right), v \in \mathcal{T}_{h}^{0}$ and $\widehat{u}^{1}$ be the pull-back (see (17)) of $\left.u\right|_{v^{\prime}}$ on the reference cell $\widehat{C}_{N}$ for a suitable $n$. We estimate as follows:

$$
\begin{aligned}
\left\|u^{1}-\mathrm{I}_{h}^{1} u^{1}\right\|_{0, t}^{2} & \lesssim\left|\operatorname{det} \mathfrak{B}_{t}\right|\left\|\mathfrak{B}_{t}\right\|^{-2}\left\|\widehat{u}^{1}-\widehat{\mathrm{I}}^{1} \widehat{u}^{1}\right\|_{0, \widehat{C}_{N}}^{2} \\
& \lesssim\left|\operatorname{det} \mathfrak{B}_{t}\right|\left\|\mathfrak{B}_{t}\right\|^{-2}\left(\left|\widehat{u}^{1}\right|_{s, \widehat{C}_{N}}^{2}+\left|\operatorname{curl} \widehat{u}^{1}\right|_{0, \widehat{C}_{N}}^{2}+\left|\operatorname{curl} \widehat{u}^{1}\right|_{s, \widehat{C}_{N}}^{2}\right) \\
& \lesssim\left(h^{2 s}\left|u^{1}\right|_{s, v^{\prime}}^{2}+h^{2}\|\operatorname{curl} u\|_{s, v^{\prime}}^{2}\right)
\end{aligned}
$$

where in the second line, we used the Deny-Lions Lemma for edge elements (see Alonso-Valli [1, Formula (5.12)] and also Monk 24]) together with the reproducing polynomial property; and in the third line we simply use (18b), (18c).

On the other hand

$$
\begin{aligned}
\left\|\operatorname{curl} u^{1}-\operatorname{curl}_{h}^{1} u^{1}\right\|_{0, t}^{2} & \lesssim\left|\operatorname{det} \mathfrak{B}_{t}\right| \int_{\widehat{C}_{N}}\left|\left(\operatorname{det} D \mathfrak{F}_{v}\right)^{-1}\left(\operatorname{curl} \widehat{u}^{1}-\widehat{\mathrm{I}}^{2} \operatorname{curl} \widehat{u}^{1}\right)\right|^{2} \\
& \lesssim\left|\operatorname{det} \mathfrak{B}_{t}\right|\left(\sup _{\hat{x} \in \widehat{C}_{N}}\left|\operatorname{det} D \mathfrak{F}_{v}(\hat{x})\right|^{-2}\right)\left|\operatorname{curl} \widehat{u}^{1}\right|_{s, \widehat{C}_{N}}^{2} \\
& \lesssim h^{2 s}\left|\operatorname{curl} u^{1}\right|_{s, v^{\prime}}^{2} .
\end{aligned}
$$

Corollary 3.3. Let $u^{1} \in \mathrm{H}_{\text {curl }}^{s}(\Gamma)$, for $s>0$, be such that $\operatorname{curl} u^{1} \in \mathrm{Y}_{h}^{2}$. Then

$$
\left\|u^{1}-\mathrm{I}_{h}^{1} u^{1}\right\|_{0} \lesssim C h^{s}\|u\|_{s} \quad 0<s<\frac{1}{2} .
$$

Proof. Let $v \in \mathcal{T}_{h}^{0}$, and $\widehat{u}^{1}$ be the pull-back of $\left.u^{1}\right|_{v^{\prime}}$ on the corresponding reference cell $\widehat{C}_{N}$. From (25), we have:

$$
\left\|u^{1}-\mathrm{I}_{h}^{1} u^{1}\right\|_{0, t}^{2} \lesssim\left(\left|\widehat{u}^{1}\right|_{s, \widehat{C}_{N}}^{2}+\left|\operatorname{curl} \widehat{u}^{1}\right|_{0, \widehat{C}_{N}}^{2}+\left|\operatorname{curl} \widehat{u}^{1}\right|_{s, \widehat{C}_{N}}^{2}\right) .
$$

Since curl $\widehat{u}^{1}$ belongs to a finite dimensional space (indeed, a space of dimension 1) and all norms are equivalent on finite dimensional spaces, (28) reduces to

$$
\left\|u^{1}-\mathrm{I}_{h}^{1} u^{1}\right\|_{0, t}^{2} \lesssim\left|\widehat{u}^{1}\right|_{s, \widehat{C}_{N}}^{2} .
$$

We conclude by using the scaling argument (18b). 
Corollary 3.4. For each $u^{0} \in \widetilde{\mathrm{X}}_{h}^{0}$, it holds that:

$$
\begin{aligned}
\left\|\mathrm{I}_{h}^{0} u^{0}\right\|_{1} & \lesssim\left\|u^{0}\right\|_{1}, \\
\left\|u^{0}-\mathrm{I}_{h}^{0} u^{0}\right\|_{0} & \lesssim h\left|u^{0}\right|_{1} .
\end{aligned}
$$

Proof. It is enough to repeat the argument used in (iii) in the proof of Theorem 3.2. In (24), $\widehat{u}^{0}$ belongs to a finite dimensional space, and we can then use the norm equivalence:

$$
\left|\widehat{u}^{0}\right|_{1+s, \widehat{C}_{N}} \lesssim\left|\widehat{u}^{0}\right|_{1, \widehat{C}_{N}} .
$$

3.2. Discrete Helmholtz-Hodge decomposition. The aim of this section is to prove stability of the discrete Helmholtz-Hodge decomposition of $\mathrm{Y}_{h}^{1}$. We suppose for simplicity that the manifold $\Gamma$ has trivial topology. The following two propositions are direct consequences of Proposition 2.5 and Corollary 3.3. Their proofs are very similar to the ones of the corresponding results for the space $\mathrm{X}_{h}^{1}$ (see, e.g., Hiptmair-Schwab [21] or Buffa et al. [13]), but we report them for the sake of completeness.

Proposition 3.5. Let $u_{\perp}^{1} \in \mathrm{Y}_{h}^{1}$ be such that $\int_{\Gamma} \operatorname{grad} \phi^{0} \cdot u_{\perp}^{1}=0 \forall \phi^{0} \in \mathrm{Y}_{h}^{0}$ and solve the following problem: Find $\varphi \in \mathrm{H}_{\mathrm{curl}}(\Gamma)$ such that:

$$
\operatorname{curl} \varphi=\operatorname{curl} u_{\perp}^{1}, \quad \operatorname{div} \varphi=0 .
$$

There exists $a \bar{s} \in\left(0, \frac{1}{2}\right)$ such that:

$$
\left\|u_{\perp}^{1}-\varphi\right\|_{0} \lesssim h^{s}\left\|\operatorname{curl} u_{\perp}^{1}\right\|_{-1+s}, \quad s \in(0, \bar{s}] .
$$

Proof. It is known that the problem defining $\varphi$ is well-posed (see, e.g., 21]), and that the solution verifies the following regularity estimate: there exists an $\bar{s}, \bar{s} \in$ $\left(0, \frac{1}{2}\right)$ such that $\varphi \in \mathrm{H}^{\bar{s}}(\Gamma)$ and

$$
\forall s \in[0, \bar{s}]:\|\varphi\|_{s} \lesssim\|\operatorname{curl} \varphi\|_{-1+s} .
$$

Thus, the interpolation operator $\mathrm{I}_{h}^{1}$ is well defined on $\varphi$ and Corollary 3.3 ensures that:

$$
\left\|\varphi-\mathrm{I}_{h}^{1} \varphi\right\|_{0} \lesssim h^{s}\|\varphi\|_{s}, \quad s \in(0, \bar{s}] .
$$

On the other hand, by construction, it holds $u_{\perp}^{1}-\mathrm{I}_{h}^{1} \varphi \in \operatorname{grad} \mathrm{Y}_{h}^{0}$. Orthogonality implies:

$$
\left\|\varphi-u_{\perp}^{1}\right\|_{0}^{2}=\int\left(\varphi-u_{\perp}^{1}\right) \cdot\left(\varphi-\mathrm{I}_{h}^{1} \varphi\right) .
$$

Combining (34), (35), (36), we obtain:

$$
\left\|\varphi-u_{\perp}^{1}\right\|_{0} \lesssim h^{s}\|\varphi\|_{s} \lesssim h^{s}\left\|\operatorname{curl} u_{\perp}^{1}\right\|_{-1+s}, \quad s \in(0, \bar{s}] .
$$

We are now ready for the following statement:

Proposition 3.6. Let $u^{1} \in \mathrm{Y}_{h}^{1}$. Then

$$
\begin{aligned}
& u^{1}=\operatorname{grad} u^{0}+u_{\perp}^{1} \quad u^{0} \in \mathrm{Y}_{h}^{0}, u_{\perp}^{1} \in \mathrm{Y}_{h}^{1}: \\
& \quad \int u^{0}=0, \quad \int \operatorname{grad} \phi^{0} \cdot u_{\perp}^{1}=0 \quad \forall \phi^{0} \in \mathrm{Y}_{h}^{0} \text { and } \\
& \text { (i) } \quad\left\|u_{\perp}^{1}\right\|_{-\frac{1}{2}+t} \lesssim\left\|\operatorname{curl} u^{1}\right\|_{-\frac{1}{2}+t} t \in\left(-\frac{1}{2}, \frac{1}{2}\right) ; \\
& \text { (ii) } \quad\left\|u^{0}\right\|_{\frac{1}{2}+t} \lesssim\left\|u^{1}\right\|_{-\frac{1}{2}+t, \text { curl }} \quad t \in\left(-\frac{1}{2}, \frac{1}{2}\right) .
\end{aligned}
$$


Proof. Let $u^{0} \in \mathrm{Y}_{h}^{0}$ be the unique solution, up to a global constant, of the problem:

$$
\int \operatorname{grad} u^{0} \cdot \operatorname{grad} \phi^{0}=\int u^{1} \cdot \operatorname{grad} \phi^{0} \quad \forall \phi^{0} \in \mathrm{Y}_{h}^{0},
$$

and $u_{\perp}^{1}=u^{1}-\operatorname{grad} u^{0}$. On the other hand, since $u^{1} \in \mathrm{H}_{\text {curl }}(\Gamma)$, it admits a continuous Helmholtz-Hodge decomposition:

$$
u^{1}=\operatorname{grad} p+\varphi, \quad p \in \mathrm{H}^{1}(\Gamma), \varphi \in \mathrm{L}^{2}(\Gamma):\left\{\begin{array}{l}
\operatorname{curl} \varphi=\operatorname{curl} u^{1} \\
\operatorname{div} \varphi=0 .
\end{array}\right.
$$

The vectorfield $\varphi$ is defined as in Proposition 3.5, By means of Corollary 3.3, $\mathrm{I}_{h}^{1} \varphi$ is well defined and it holds: $\left\|\mathrm{I}_{h}^{1} \varphi\right\|_{0} \lesssim\|\varphi\|_{s}, s>0$. By orthogonality and Proposition 2.5. it follows easily that $\int\left|u_{\perp}^{1}\right|^{2}=\int u_{\perp}^{1} \cdot I_{h}^{1}(\varphi)$. Thus, we deduce that, for any fixed $s>0$, it holds that:

$$
\left\|u_{\perp}^{1}\right\|_{0} \lesssim\|\varphi\|_{s} \lesssim\left\|\operatorname{curl} u^{1}\right\|_{-1+s} .
$$

This implies that (37)(i) holds true. We deduce (37)(ii) by the difference and recall that grad: $\mathrm{H}^{\frac{1}{2}+t}(\Gamma) \rightarrow \mathrm{H}^{-\frac{1}{2}+t}(\Gamma)$ is continuous and has closed range for each $t \in\left(-\frac{1}{2}, \frac{1}{2}\right)$.

3.3. LBB Inf-Sup condition. We now show that the spaces $Y_{h}^{i}$ and $X_{h}^{2-i}$ are dual to each other in the sense that the $\mathrm{L}^{2}$ dualities restricted to these spaces satisfy uniform LBB Inf-Sup conditions in appropriate norms. These results are proved under the assumption that the family of meshes $\left(\mathcal{T}_{h}\right)$ is quasi-uniform. In addition, for Proposition 3.11 we need a combinatorial non-degeneracy condition stated at the end of its proof. We first prove dualities in $\mathrm{L}^{2}$ norms based on a matrix argument and then extend the results by more functional analytic techniques.

We will use the following fact on diagonally dominant matrices:

Lemma 3.7. Suppose $\left(a^{i j}\right)$ is a square real matrix with non-negative diagonal coefficients, which is diagonally dominant with respect both to rows and columns in the sense that for some $\delta \leq 1$ we have:

$$
\delta a^{i i} \geq \sum_{j \neq i}\left|a^{i j}\right| \quad \text { and } \quad \delta a^{j j} \geq \sum_{i \neq j}\left|a^{i j}\right| .
$$

Then for all tuples $\left(u_{i}\right)$ we have:

$$
(1-\delta) \sum_{i} a^{i i}\left|u_{i}\right|^{2} \leq \sum_{i j} a^{i j} u_{i} u_{j} \leq(1+\delta) \sum_{i} a^{i i}\left|u_{i}\right|^{2} .
$$

Proof. The study of Gersgorin discs (see, e.g., Horn-Johnson 22 p. 349) shows that a real symmetric matrix which is diagonally dominant and has non-negative diagonal elements is positive semi-definite.

Let $d$ denote the diagonal of $a$. The above remark yields:

$$
\left(a+a^{\mathrm{T}}\right) / 2-(1-\delta) d \geq 0 \text { and }(1+\delta) d-\left(a+a^{\mathrm{T}}\right) / 2 \geq 0 .
$$

This proves the lemma.

For each vertex $v$ let $N_{v}$ be the number of triangles $t \in \mathcal{T}_{h}^{2}$ such that $v \in|t|$. Let $N^{\text {max }}$ be largest such number. 
$\mathrm{L}^{2}$ duality on $Y_{h}^{0} \times X_{h}^{2}$. The following proposition holds true.

Proposition 3.8. For each pair of triangles $s, t \in \mathcal{T}_{h}^{2}$ put $a^{s t}=\int \mu_{s}^{0} \lambda_{t}^{2}$. Then there is a $\delta<1$ depending only on $N^{\max }$ for which the matrix $\left(a^{\text {st }}\right)$ satisfies (38).

Proof. By definition, for each triangle $t, \lambda_{t}^{2}$ is the constant function on $|t|$ whose integral is 1. It follows that on each of the six triangles of the barycentric refinement contained in $t$, the integral of $\lambda_{t}^{2}$ is $1 / 6$. The integral of an affine function times $\lambda_{t}^{2}$ on such a triangle is therefore $1 / 18$ of the sum of its values at its vertices.

Fix a triangle $t \in \mathcal{T}_{h}^{2}$. We denote by $u, v$ and $w$ its vertices.

We first compute $\int \mu_{t}^{0} \lambda_{t}^{2}$. Using the above remark on the six subtriangles of $t$ gives:

$$
\begin{aligned}
a^{t t}=\int \mu_{t}^{0} \lambda_{t}^{2} & =(1 / 18)\left(6+6(1 / 2)+2 / N_{u}+2 / N_{v}+2 / N_{w}\right) \\
& =1 / 2+\left(1 / N_{u}+1 / N_{v}+1 / N_{w}\right) / 9 .
\end{aligned}
$$

Next we compute the terms on the columns of the matrix $a$. They're all positive, and since $\mu^{0}$ is a partition of unity, the sum of the column terms is the integral of $\lambda_{t}^{0}$ which is 1 . This yields:

$$
\sum_{s \neq t}\left|a^{s t}\right|=1 / 2-\left(1 / N_{u}+1 / N_{v}+1 / N_{w}\right) / 9 .
$$

For the row we also remark that all terms are positive. Using the above remark repeatedly we obtain:

$$
\sum_{s} \int \mu_{t}^{0} \lambda_{s}^{2}=1
$$

Therefore it suffices to take $\delta$ defined by:

$$
\delta=\max \frac{1 / 2-\left(1 / N_{u}+1 / N_{v}+1 / N_{w}\right) / 9}{1 / 2+\left(1 / N_{u}+1 / N_{v}+1 / N_{w}\right) / 9} \leq \frac{1-2 /\left(3 N^{\max }\right)}{1+2 /\left(3 N^{\max }\right)}<1 .
$$

This concludes the proof.

Proposition 3.9. Suppose that the family of meshes is quasi-uniform. There is $C>0$ such that for all $h$ :

$$
\inf _{u \in X_{h}^{2}} \sup _{v \in Y_{h}^{0}} \frac{\int u v}{\|u\|_{0}\|v\|_{0}} \geq 1 / C .
$$

Proof. Let $\Lambda_{h}: X_{h}^{2} \rightarrow Y_{h}^{0}$ be the map defined by:

$$
\Lambda_{h}: \sum_{s} u_{s} \lambda_{s}^{2} \rightarrow 1 / h^{2} \sum_{s} u_{s} \mu_{s}^{0} .
$$

The map $\Lambda_{h}$ can be written uniquely as $\Lambda_{h}=I_{h}^{0} \Theta_{h}$ where $I_{h}^{0}: X_{h}^{2} \rightarrow Y_{h}^{0}$ is the interpolation operator defined by the degrees of freedom, and $\Theta_{h}: X_{h}^{2} \rightarrow X_{h}^{2}$ is diagonal in the basis $\lambda^{2}$. Both $I_{h}^{0}: X_{h}^{2} \rightarrow Y_{h}^{0}$ and $\Theta_{h}: X_{h}^{2} \rightarrow X_{h}^{2}$ are stable in the $\mathrm{L}^{2}$ norm, uniformly with respect to $h$ (the first because of a finite dimensionality argument on the reference cells and the second because by quasi-uniformity the 
areas of the triangles are equivalent to $h^{2}$ ). For $u=\sum_{s} u_{s} \lambda_{s}^{2}$ we can write:

$$
\begin{aligned}
\int u \Lambda_{h} u & =1 / h^{2} \sum_{s t} \int \lambda_{s}^{2} \mu_{t}^{0} u_{s} u_{t} \\
& \geq 1 /\left(C h^{2}\right) \sum_{s} u_{s}^{2} \\
& \geq 1 / C\|u\|_{0}^{2} \\
& \geq 1 / C\|u\|_{0}\left\|\Lambda_{h} u\right\|_{0} .
\end{aligned}
$$

This concludes the proof.

Proposition 3.10. Suppose that the family of meshes is quasi-uniform. There is $C>0$ such that for all $s \in[0,1]$ and all $h$ :

$$
\inf _{u \in X_{h}^{2}} \sup _{v \in Y_{h}^{0}} \frac{\int u v}{\|u\|_{-s}\|v\|_{s}} \geq 1 / C .
$$

Proof. Let $P_{h}$ be the Ritz-Galerkin projector onto $Y_{h}^{0}$, associated with the $\mathrm{L}^{2}$ duality on $Y_{h}^{0} \times X_{h}^{2}$. By the preceding proposition it is stable in the $\mathrm{L}^{2}$ norm, i.e., there is $C>0$ such that:

$$
\left\|P_{h} u\right\|_{0} \lesssim\|u\|_{0}
$$

By standard interpolation arguments it is enough to prove that it is also stable in the $\mathrm{H}^{1}$ norm for the proposition to hold.

Let $Q_{h}$ be the projector onto $Y_{h}^{0}$ constructed as $Q_{h}=I_{h}^{0} R_{h}$ where $I_{h}^{0}: \widetilde{X}_{h}^{0} \rightarrow Y_{h}^{0}$ is the interpolator and $R_{h}: \mathrm{L}^{2}(\Gamma) \rightarrow \widetilde{X}_{h}^{0}$ is the (standard) $\mathrm{L}^{2}$ projection. Then $Q_{h}$ satisfies:

$$
\begin{aligned}
\left\|Q_{h} u\right\|_{1} & \lesssim\|u\|_{1}, \\
\left\|u-Q_{h} u\right\|_{0} & \lesssim h\|u\|_{1},
\end{aligned}
$$

since these properties hold for both $I_{h}^{0}$ and $R_{h}$ (see Corollary 3.4).

Now we can simply write:

$$
\begin{aligned}
\left\|P_{h} u\right\|_{1} & \leq\left\|P_{h} u-Q_{h} u\right\|_{1}+\left\|Q_{h} u\right\|_{1} \\
& \lesssim h^{-1}\left\|P_{h}\left(u-Q_{h} u\right)\right\|_{0}+\left\|Q_{h} u\right\|_{1} \\
& \lesssim h^{-1}\left\|\left(u-Q_{h} u\right)\right\|_{0}+\left\|Q_{h} u\right\|_{1} \\
& \lesssim\|u\|_{1} .
\end{aligned}
$$

This concludes the proof.

$\mathrm{L}^{2}$ duality on $Y_{h}^{2} \times X_{h}^{0}$. This duality is more well-known at least to the extent that $Y_{h}^{2}$ is close to the space of piecewise constants on the Voronoi dual mesh, but we sketch a proof nevertheless, following the lines of the previous case.

Proposition 3.11. For each pair of vertices $s, t \in \mathcal{T}_{h}^{0}$ put $a^{s t}=\int \mu_{s}^{2} \lambda_{t}^{0}$. Under a mild local non-degeneracy condition given at the end the proof, the matrix $\left(a^{s t}\right)$ satisfies (38).

Proof. First we check that, since the integral of $\mu_{s}^{2}$ times an affine function of $\mathcal{T}_{h}^{\prime}$ on a triangle containing $s$ is $1 /\left(6 N_{s}\right)$ of the sum of its values at the vertices, we have:

$$
\int \mu_{s}^{2} \lambda_{s}^{0}=(1+1 / 2+1 / 3) / 3=11 / 18 .
$$


Moreover, $\lambda^{0}$ is a partition of unity giving :

$$
\sum_{t} \mu_{s}^{2} \lambda_{t}^{0}=1
$$

We can also compute the sum of the column terms (all of which are positive):

$$
\begin{aligned}
\sum_{t} \mu_{s}^{2} \lambda_{t}^{0} & =11 / 18+((1 / 3+1 / 3+1 / 2) / 3)\left(\sum_{t} 1 / N_{t}\right) \\
& =11 / 18+(7 / 18)\left(\sum_{t} 1 / N_{t}\right)
\end{aligned}
$$

where the last sum is taken over the $N_{s}$ vertices $t$ such that $\{s, t\} \in \mathcal{T}_{h}^{1}$.

It therefore suffices that the family of meshes is such that for some $\delta^{\prime}<1$ we have for each $h$ and $s \in \mathcal{T}_{h}^{0}$ :

$$
\sum_{t \in \mathcal{T}_{h}^{0}:\{s, t\} \in \mathcal{T}_{h}^{1}} 1 / N_{t} \leq \delta^{\prime}(11 / 7)
$$

which is easy to achieve.

Proposition 3.12. Suppose that the family of meshes is quasi-uniform. There is $C>0$ such that for all $h$ :

$$
\inf _{u \in X_{h}^{0}} \sup _{v \in Y_{h}^{2}} \frac{\int u v}{\|u\|_{0}\|v\|_{0}} \geq 1 / C .
$$

Proof. Let $\Lambda_{h}: X_{h}^{0} \rightarrow Y_{h}^{2}$ be the map defined by:

$$
\Lambda_{h}: \sum_{s} u_{s} \lambda_{s}^{0} \rightarrow h^{2} \sum_{s} u_{s} \mu_{s}^{2}
$$

The map $\Lambda_{h}$ is stable in $\mathrm{L}^{2}$ by the quasi-uniformity hypothesis, since the mass matrix on $X_{h}^{0}$ in the basis $\lambda^{0}$ is uniformly strictly diagonally dominant (see, e.g., Brenner-Scott [6]). For $u=\sum_{s} u_{s} \lambda_{s}^{0}$ we can repeat the preceding argument:

$$
\begin{aligned}
\int u \Lambda_{h} u & =h^{2} \sum_{s t} \int \lambda_{s}^{2} \mu_{t}^{0} u_{s} u_{t} \\
& \geq h^{2} / C \sum_{s} u_{s}^{2} \\
& \geq 1 / C\|u\|_{0}^{2} \\
& \geq 1 / C\|u\|_{0}\left\|\Lambda_{h} u\right\|_{0} .
\end{aligned}
$$

This concludes the proof.

Proposition 3.13. Suppose that the family of meshes is quasi-uniform. There is $C>0$ such that for all $s \in[0,1]$ and all $h$ :

$$
\inf _{u \in X_{h}^{0}} \sup _{v \in Y_{h}^{2}} \frac{\int u v}{\|u\|_{s}\|v\|_{-s}} \geq 1 / C .
$$

Proof. It is enough to prove that the Ritz-Galerkin projector onto $X_{h}^{0}$ with respect to $\mathrm{L}^{2}$ duality on $X_{h}^{0} \times Y_{h}^{2}$ is stable in the $\mathrm{H}^{1}$ norm. This can be proved as in the case of the projector onto $Y_{h}^{0}$ relative to $X_{h}^{2} \times Y_{h}^{0}$ (see the proof of Proposition (3.10). 
$\mathrm{L}^{2}$ duality on $Y_{h}^{1} \times X_{h}^{1}$. In fact the matrix relative to our choices of basis is not diagonally dominant, as shown in the appendix. However using discrete Hodge decompositions we can deduce Inf-Sup conditions for the most important pairs of norms. Indeed, following Buffa-Ciarlet [11] and Buffa [8, we know that the $\mathrm{L}^{2}$ scalar product extends to a continuous non-degenerate duality pairing between $\mathrm{H}_{\text {div }}^{-\frac{1}{2}+s}(\Gamma) \times \mathrm{H}_{\text {curl }}^{-\frac{1}{2}-s}(\Gamma), s \in\left(-\frac{1}{2}, \frac{1}{2}\right)$, i.e. there exists a constant $C>0$ such that:

$$
\inf _{u \in \mathrm{H}_{\text {div }}^{-1 / 2+s}(\Gamma)} \sup _{v \in \mathrm{H}_{\text {curl }}^{-1 / 2-s}(\Gamma)} \frac{\int_{\Gamma} u \cdot v}{\|u\|_{-\frac{1}{2}+s, \operatorname{div}}\|v\|_{-\frac{1}{2}-s, \text { curl }}} \geq 1 / C,
$$

and we shall now prove the discrete analogue. Again, for simplicity we suppose that the topology of $\Gamma$ is trivial in the sense that curl-free (resp. divergence-free) vector fields are gradients (resp. curls).

Proposition 3.14. Suppose that the family of meshes is quasi-uniform. Then for each $s \in\left(-\frac{1}{2}, \frac{1}{2}\right)$ there is $C>0$ such that for all $h$ :

$$
\inf _{u \in X_{h}^{1}} \sup _{v \in Y_{h}^{1}} \frac{\int u \cdot v}{\|u\|_{-\frac{1}{2}+s, \text { div }}\|v\|_{-\frac{1}{2}-s, \text { curl }}} \geq 1 / C .
$$

Proof. Fix $s \in\left(-\frac{1}{2}, \frac{1}{2}\right)$ and a $u \in X_{h}^{1}$. Write $u \in X_{h}^{1}$ in the form $u=\operatorname{curl} p+u_{\perp}$ with $p \in X_{h}^{0}$ and $\forall p^{\prime} \in X_{h}^{0} \int u_{\perp} \cdot \operatorname{curl} p^{\prime}=0$. Moreover, following Hiptmair-Schwab 21]:

(1) there exists a curl free $\phi$ such that $\left\|\phi-u_{\perp}\right\|_{-\frac{1}{2}+s, \text { div }} \lesssim \mu_{h}\|u\|_{-\frac{1}{2}+s \text {, div }}$, for a given sequence $\mu_{h}$ converging to 0 as $h \rightarrow 0$;

(2) for all $s \in\left(-\frac{1}{2}, \frac{1}{2}\right)$, it holds that:

$$
\left\|u_{\perp}\right\|_{-\frac{1}{2}+s} \lesssim\|\operatorname{div} u\|_{-\frac{1}{2}+s} .
$$

Similarly write $v \in Y_{h}^{1}$ in the form $v=\operatorname{grad} q+v_{\perp}$ with $q \in Y_{h}^{0}$ and $\forall q^{\prime} \in$ $X_{h}^{0} \int v_{\perp} \cdot \operatorname{grad} q^{\prime}=0$. Proposition 3.5 gives a divergence free $\psi$ such that $\| \psi-$ $v_{\perp}\left\|_{-\frac{1}{2}-s, \text { curl }} \leq \nu_{h}\right\| v \|_{-\frac{1}{2}-s, \text { curl }}$, for a given sequence $\nu_{h}$ converging to 0 as $h \rightarrow 0$.

We have:

$$
\int u \cdot v=\int p \operatorname{curl} v_{\perp}-\int \operatorname{div} u_{\perp} q+\int u_{\perp} \cdot v_{\perp} .
$$

Moreover, since $\int \phi \cdot \psi=0$ we have:

$$
\begin{aligned}
\left|\int u_{\perp} \cdot v_{\perp}\right| & =\left|\int\left(u_{\perp}-\phi\right) \cdot \psi+\phi \cdot\left(v_{\perp}-\psi\right)+\left(u_{\perp}-\phi\right) \cdot\left(v_{\perp}-\psi\right)\right| \\
& \lesssim \max \left\{\mu_{h}, \nu_{h}\right\}\|u\|_{-\frac{1}{2}+s, \text { div }}\|v\|_{-\frac{1}{2}-s, \text { curl }} \cdot
\end{aligned}
$$

The Inf-Sup conditions (67) and (52) proved for the scalar cases in $\mathrm{H}^{-1 / 2 \pm s} \times$ $\mathrm{H}^{1 / 2 \mp s}$ norms, together with (37) (i) and (69), give the Inf-Sup condition (68) for $h$ small enough. This method of proof extends to non-trivial topologies.

Next we show that, for large $h$, degeneracy cannot occur in trivial topology. This uses Proposition 2.5 repeatedly. Given $u \in X_{h}^{1}$ we suppose that quantity (70) is zero for all $v \in Y_{h}^{1}$. First, taking $v$ to be of the form $v=\operatorname{grad} q$ (so that $v_{\perp}=0$ ) with $q \in Y_{h}^{0}$ shows that $\operatorname{div} u=0$. Therefore $u$ is in the form $u=\operatorname{curl} p$ for some $p \in X_{h}^{0}$ with 0 mean value. Given the range of curl $: Y_{h}^{1} \rightarrow Y_{h}^{2}$, such a $p$ must be 0 , which shows that $u$ is zero. 


\section{Remarks, Extensions And APPlications}

4.1. In the language of differential forms. The results of this paper can be cast in the language of differential forms. This is achieved through the standard correspondence of various kinds of forms with functions and vectorfields. Let $g$ be the Riemannian metric on $\Gamma$ and $\omega$ the canonical volume-form on $\Gamma$ associated with $g$ and the orientation of $\Gamma$. A function $u$ on $\Gamma$ is a 0 -form but also gives rise to the 2-form $u \omega$, and a vectorfield $u$ corresponds to the 1-forms $\omega(u, \cdot)$ and $g(u, \cdot)$. Departing from the definitions preceding Proposition 2.4 we let $\Omega^{i}(\Gamma)$ denote the space of $i$-forms on $\Gamma$ obtained as follows :

- $\Omega^{2}(\Gamma)$ is the range of the map $u \mapsto u \omega$ on $\mathrm{H}^{-1 / 2}(\Gamma)$.

- $\Omega^{1}(\Gamma)$ is the range of the map $u \mapsto \omega(u, \cdot)$ on $\mathrm{H}_{\text {div }}^{-1 / 2}(\Gamma)$, or alternatively as the range of the map $u \mapsto g(u, \cdot)$ on $\mathrm{H}_{\text {rot }}^{-1 / 2}(\Gamma)$.

- $\Omega^{0}(\Gamma)$ is the space $\mathrm{H}^{1 / 2}(\Gamma)$.

The equality of the two definitions given for $\Omega^{1}(\Gamma)$ is based on the identity

$$
\omega(u \times n, \cdot)=g(u, \cdot)
$$

and the fact that $u \mapsto u \times n$ provides an isomorphism $\mathrm{H}_{\text {rot }}^{-1 / 2}(\Gamma) \rightarrow \mathrm{H}_{\operatorname{div}}^{-1 / 2}(\Gamma)$. We also assume that $\Omega^{i}(\Gamma)$ has been equipped with the norm, denoted $\mathrm{H}_{\mathrm{d}}^{-1 / 2}$, which makes the defining maps isometries.

Departing slightly from the notations of the previous sections we interpret $X_{h}^{i}$ as a space of $i$-forms, for each $i \in\{0,1,2\}$, using the maps defining the spaces $\Omega^{i}(\Gamma)$. This interpretation goes back to Bossavit [5. The bases chosen for these spaces are also the ones obtained by mapping the preceding ones. The spaces $X_{h}^{i}$ form a complex under the exterior derivative d.

This procedure associates a complex $X_{h}^{\bullet}$ to any simplicial complex $\mathcal{T}_{h}$ on $\Gamma$, together with a choice of basis for each of the spaces $X_{h}^{i}$. In particular, given $\mathcal{T}_{h}$ we obtain $X_{h}^{\bullet}$ and $\lambda^{\bullet}$, as well as a complex $X_{h}^{\prime \bullet}$ and bases $\lambda^{\prime \bullet}$ associated with the barycentric refinement $\mathcal{T}_{h}^{\prime}$ of $\mathcal{T}_{h}$. The complex $Y_{h}^{\bullet}$ is then constructed as a subcomplex of $X_{h}^{\prime \bullet}$, such that the space $Y_{h}^{i}$ is equipped with a basis $\mu^{i}$ whose elements are linear combinations of the basis-elements $\lambda^{\prime i}$ with the same coefficients as before.

The previous results straightforwardly carry over to this setting. Recall that integration is a linear form on the space of 2 -forms. The bilinear form $(u, v) \mapsto$ $\int u \wedge v$ defined on $i$ and $(2-i)$ forms $u$ and $v$ is non-degenerate and called Hodge duality. In the continuous case non-degeneracy can be deduced from the existence of the Hodge star operator (see Taylor [28] p. 355), whose definition we now recall. It is the map $*$ which to any $i$-form $u$ associates the $(2-i)$-form $* u$ defined pointwise by the property that:

$$
v \wedge * u=(v \cdot u) \omega
$$

for each $i$-form $v$, where the notation - is used to denote the scalar product on alternating forms induced by the metric $g$.

Much of this paper is motivated by the following discrete non-degeneracy property. 
Proposition 4.1. Suppose that the family of meshes is quasi-uniform. For each $i$ there is $C>0$ such that for all $h$ :

$$
\inf _{u \in X_{h}^{i}} \sup _{v \in Y_{h}^{2-i}} \frac{\int u \wedge v}{\|u\|_{\mathrm{H}_{\mathrm{d}}^{-1 / 2}}\|v\|_{\mathrm{H}_{\mathrm{d}}^{-1 / 2}}} \geq 1 / C .
$$

Proof. For the case $i=1$ we remark that when $u$ and $v$ are two vectorfields we have:

$$
\omega(u, \cdot) \wedge g(v, \cdot)=-g(u, v) \omega .
$$

The present formulation is therefore just a reformulation of the vector case. For $i=0$ and $i=2$ the translations are even more direct.

This leads to the definition of a discrete Hodge star operator. We introduce the maps $*_{h}: X_{h}^{i} \rightarrow Y_{h}^{2-i}$ which to any $u \in X_{h}^{i}$ associates $*_{h} u \in Y_{h}^{2-i}$ defined by:

$$
\int v \wedge *_{h} u=\int(v \cdot u) \omega
$$

for all $v \in X_{h}^{i}$. They are well defined isomorphims by Proposition 4.1. Contrary to the continuous Hodge star, the discrete ones are non-local (do not in general preserve supports).

The formal adjoint $\delta$ of $\mathrm{d}$ is defined by the property that for all $i$-forms $u$ and all $(i-1)$-forms $v$ we have:

$$
\int \delta u \cdot v=\int u \cdot \mathrm{d} v
$$

Similarly one can define the formal adjoint $\delta_{h}: X_{h}^{i} \rightarrow X_{h}^{i-1}$ of d $: X_{h}^{i-1} \rightarrow X_{h}^{i}$.

We then have, for all $u \in X_{h}^{i}$ and all $v \in X_{h}^{i-1}$ :

$$
\begin{aligned}
\int \delta_{h} u \cdot v & =\int \mathrm{d} v \cdot u=\int \mathrm{d} v \wedge *_{h} u \\
& =(-1)^{i-1} \int v \wedge \mathrm{d} *_{h} u=(-1)^{i-1} \int v \wedge *_{h}\left(*_{h}\right)^{-1} \mathrm{~d} *_{h} u \\
& =(-1)^{i-1} \int\left(*_{h}\right)^{-1} \mathrm{~d} *_{h} u \cdot v,
\end{aligned}
$$

which shows that, on $i$-forms, $\delta_{h}=(-1)^{i-1}\left(*_{h}\right)^{-1} \mathrm{~d} *_{h}$ which is a discrete analogue of the fact that, up to signs, $\delta$ equals $* \mathrm{~d} *$, given that $*$ is its own inverse up to sign (28 p. 356). In other words we have just showed that the following diagram commutes:

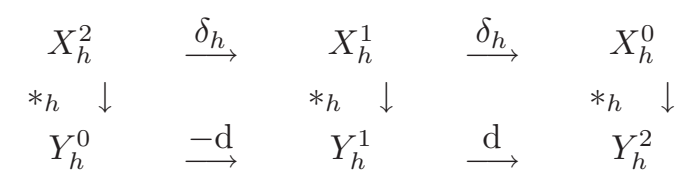

4.2. Extension to open surfaces. The aim of this section is to extend the previous definitions and results to the case of open orientable polyhedral manifolds. Then let $\gamma$ denote such a manifold and $\partial \Gamma$ its boundary.

All the notations in this section will be consistent with those introduced previously. Moreover, every time that we add a subscript $(\cdot)_{0}$ to spaces, we mean the closure of regular compactly supported functions with respect to the topology 
of the space, and we will use the superindex $(\cdot)^{b}$ to denote objects related to the boundary $\partial \Gamma$.

We are given a complex $\left(X_{h}^{0}, X_{h}^{1}, X_{h}^{2}\right)$ based on Raviart-Thomas divergence conforming vector fields satisfying homogeneous boundary conditions. It is defined by:

$$
\begin{array}{lll}
X_{h}^{0}=\left\{u \in \mathrm{H}_{0}^{1}(\Gamma)\right. & : \forall t \in \mathcal{T}_{h}^{2} & \left.\left.u\right|_{t} \in \mathcal{P}_{1}\right\}, \\
X_{h}^{1}=\left\{u \in \mathrm{H}_{\mathrm{div}, 0}(\Gamma): \forall t \in \mathcal{T}_{h}^{2}\right. & \left.\left.u\right|_{t} \in \mathrm{RT}_{0}\right\}, \\
X_{h}^{2}=\left\{u \in \mathrm{L}^{2}(\Gamma) \quad: \forall t \in \mathcal{T}_{h}^{2}\right. & \left.\left.u\right|_{t} \in \mathcal{P}_{0}\right\} .
\end{array}
$$

We then construct a suitable complex $\mathrm{Y}_{h}^{\bullet}$ such that the dualities on $\mathrm{Y}_{h}^{i} \times \mathrm{X}_{h}^{2-i}$, $i=0,1,2$, are non-degenerate. Moreover, the complex $\mathrm{Y}_{h}^{\bullet}$ is built as a sub-complex of $\widetilde{\mathrm{X}}_{h}^{\bullet}$, which is defined exactly as in (3). We proceed in a constructive way by modifying the basis functions introduced in Section 2 in order to take into account the boundary $\partial \Gamma$.

Note that hereafter we construct one particular complex, but others may be constructed enjoying different properties, depending on their intended use. In what follows, our construction is mainly driven by the requirement that the dualities on $\mathrm{Y}_{h}^{i} \times \mathrm{X}_{h}^{2-i}$ are non-degenerate.

(i) Boundary basis functions for $\mathrm{Y}_{h}^{0}$. Let $t \in \mathcal{T}_{h}^{2}$ be a triangle interacting with the boundary $\partial \Gamma$. Two situations may occur: (i) $t \cap \partial \Gamma \in \mathcal{T}_{h}^{1}$, i.e., $t$ shares an edge with the boundary, then $\mu_{t}^{0, b} \in \widetilde{\mathrm{X}}_{h}^{0}$ have the coefficients shown in Figure 4(a); (ii) $t \cap \partial \Gamma \in \mathcal{T}_{h}^{0}$, i.e., $t$ shares a vertex with the boundary, then $\mu_{t}^{0, b} \in \widetilde{\mathrm{X}}_{h}^{0}$ have the coefficients shown in Figure $4(\mathrm{~b})$.

Thus, we set

$$
\mathrm{Y}_{h}^{0}=\operatorname{span}\left\{\left(\mu_{t}^{0}, t \in \mathcal{T}_{h}^{2}: t \cap \partial \Gamma=\emptyset\right),\left(\mu_{t}^{0, b}, t \in \mathcal{T}_{h}^{2}: t \cap \partial \Gamma \neq \emptyset\right)\right\} .
$$

It is a matter of trivial checking that this basis is a partition of unity.

(ii) Boundary basis functions for $\mathrm{Y}_{h}^{1}$. We associate a basis function only with those edges $e \in \mathcal{T}_{h}^{1}$ such that $e \cap \partial \Gamma \in \mathcal{T}_{h}^{0}$, i.e., which share a vertex with the boundary. Let $v \in \mathcal{T}_{h}^{0}$ be on $\partial \Gamma$ and $m_{v}+1$ the number of triangles $t \in \mathcal{T}_{h}^{2}$ sharing $v$ as a vertex. We number these triangles as $t_{0}, \ldots, t_{m_{v}}$ turning around $v$ in a counterclockwise sense. Accordingly we number edges $e$ such that $|e| \cap \partial \Gamma=|v|$ as $e_{1}, e_{2}, \ldots, e_{m_{v}-1}$ and denote by $w_{i}$ the other vertex of $e_{i}$. We suppose each $e_{i}$ to be oriented from $w_{i}$ to $v$. The boundary basis function $\mu_{e_{i}}^{1, b}$ associated with $e_{i}$ is defined as:

$$
\mu_{e_{i}}^{1, b}=\left\{\begin{array}{lr}
\mu_{e_{i}}^{1} & \text { on } w_{i}^{\prime}, \\
\sum_{j=0}^{i-1} \operatorname{grad} \mu_{t_{j}}^{0, b} & \text { on } v^{\prime} .
\end{array}\right.
$$

Note that the construction does not depend upon the orientation or the numbering we have chosen since $\sum_{j=0}^{i-1} \operatorname{grad} \mu_{t_{j}}^{0, b}=-\sum_{j=i}^{m_{v}} \operatorname{grad} \mu_{t_{j}}^{0, b}$ on $v^{\prime}$, being true that $\sum_{j=0}^{m_{v}} \mu_{t_{j}}^{0, b}=1$ on $v^{\prime}$.

Thus, we set

$$
\mathrm{Y}_{h}^{1}=\operatorname{span}\left\{\left(\mu_{e}^{1}, e \in \mathcal{T}_{h}^{1}: e \cap \partial \Gamma=\emptyset\right),\left(\mu_{e}^{1, b}, e \in \mathcal{T}_{h}^{1}: t \cap \partial \Gamma \in \mathcal{T}_{h}^{0}\right)\right\}
$$

(iii) Characterization of $\mathrm{Y}_{h}^{2}$. We do not add any boundary term to the space $\mathrm{Y}_{h}^{2}$, i.e., $\mathrm{Y}_{h}^{2}=\operatorname{span}\left\{\mu_{v}^{2}, v \in \mathcal{T}_{h}^{0}: v \notin \partial \Gamma\right\}$. 


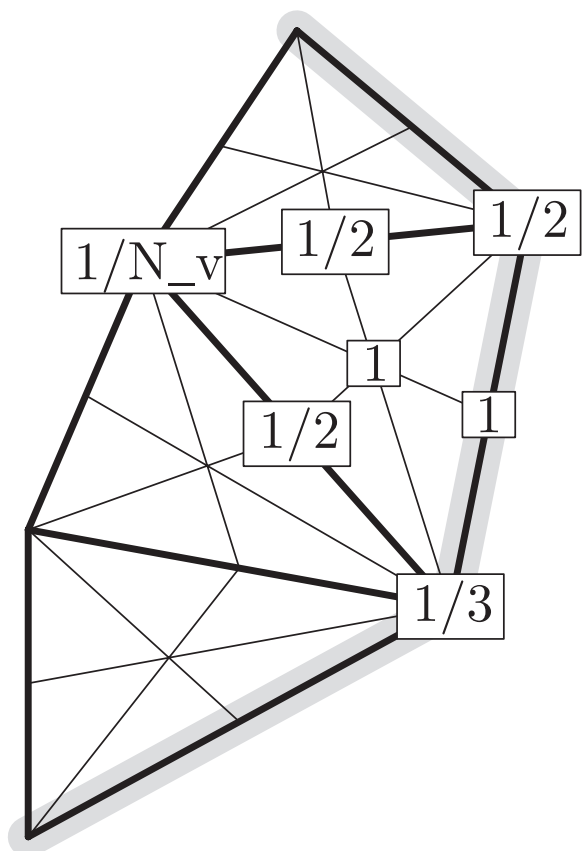

(a) Case $t \cap \partial \Gamma \in \mathcal{T}_{h}^{1}$.

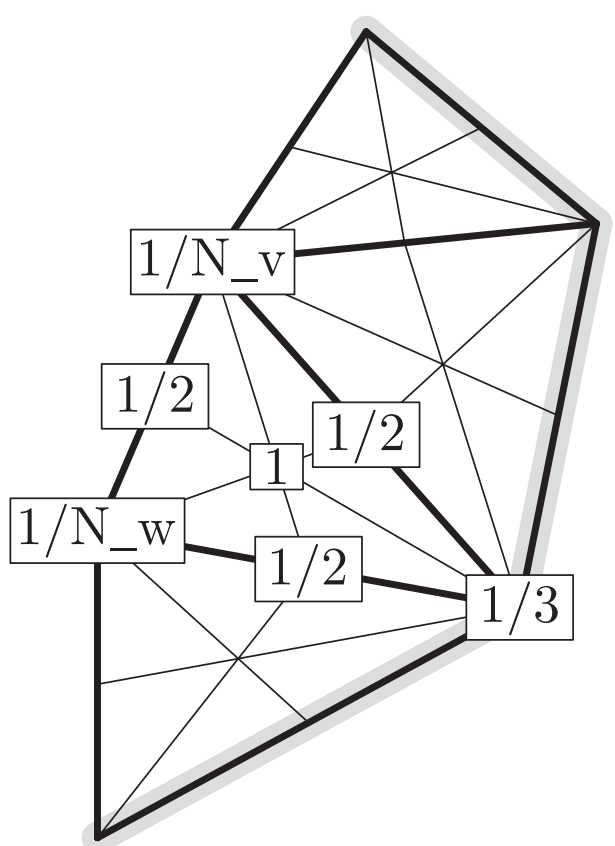

(b) Case $t \cap \partial \Gamma \in \mathcal{T}_{h}^{0}$.

Figure 4. The two types of boundary basis element for $Y_{h}^{0}$ expressed in the basis of $\widetilde{X}_{h}^{0}$. The shaded gray region corresponds to the boundary $\partial \Gamma . N_{v}$ and $N_{w}$ stand for the number of triangles sharing the nodes $v$ and $w$, respectively.

Now, we list a series of results which are basically a repetition of the ones proved in Section 2 and Section 3 . We will not repeat proofs when they are similar to the ones already presented.

Proposition 4.2 (Analogue of Proposition 2.5). In the following complex, the cohomology groups have the "right" dimension:

$$
0 \longrightarrow \mathrm{Y}_{h}^{0} \stackrel{\operatorname{grad}}{\longrightarrow} \mathrm{Y}_{h}^{1} \stackrel{\operatorname{curl}}{\longrightarrow} \mathrm{Y}_{h}^{2} \longrightarrow 0 .
$$

More precisely, for a connected manifold, for the first cohomology group an element of $Y_{h}^{0}$ has gradient 0 iff it is constant, whereas for the last cohomology group any element of $Y_{h}^{2}$ is the curl of an element of $Y_{h}^{1}$.

As far as the metric properties are concerned, we adopt the notation of Section 3. Remark that, in the case of open manifolds some attention has to be devoted to the definition of Sobolev spaces with negative regularity exponent. Hereafter, we adopt the following notation: $\mathrm{H}^{-s}(\Gamma)=\left(\mathrm{H}_{0}^{s}(\Gamma)\right)^{\prime}, s \in(0,3 / 2)$. For the singular exponents $s= \pm \frac{1}{2}$, we adopt the notation of Lions-Magenes [23] and set $\mathrm{H}_{00}^{-\frac{1}{2}}(\Gamma)=$ $\left(H_{00}^{\frac{1}{2}}(\Gamma)\right)^{\prime}$. These spaces are endowed with their standard norms $\|\cdot\|_{\frac{1}{2}, 00}$ and $\|\cdot\|_{-\frac{1}{2}, 00}$, respectively. In the same way, a singular index appears in the definition 
of $\mathrm{H}_{\text {curl }}^{s}(\Gamma)$, when $s=-\frac{1}{2}$. As in Buffa-Ciarlet [12, Sec. 6], we set:

$$
\mathrm{H}_{\text {curl }, 00}^{-\frac{1}{2}}(\Gamma)=\left\{u \in \mathrm{H}_{00}^{-\frac{1}{2}}(\Gamma): \operatorname{curl} u \in \mathrm{H}_{00}^{-\frac{1}{2}}(\Gamma)\right\},
$$

endowed with the corresponding graph norm $\|\cdot\|_{\frac{1}{2}, \text { curl,00 }}$.

We start by proving the following error estimates.

Theorem 4.3 (Analogue of Theorem 3.2 ). Let $0<s<1 / 2$. For any $u^{0} \in \mathrm{H}^{1+s}(\Gamma)$, $u^{1} \in \mathrm{H}_{\text {curl }}^{s}(\Gamma), u^{2} \in \mathrm{H}^{s}(\Gamma)$, it holds that

$$
\begin{aligned}
\left\|u^{0}-\mathrm{I}_{h}^{0} u^{0}\right\|_{1}+h^{-1}\left\|u^{0}-\mathrm{I}_{h}^{0} u^{0}\right\|_{0} & \lesssim h^{s}\left\|u^{0}\right\|_{s+1}, \\
\left\|u^{1}-\mathrm{I}_{h}^{1} u^{1}\right\|_{0, \text { curl }} & \lesssim h^{s}\left\|u^{1}\right\|_{s, \text { curl }}, \\
\left\|u^{2}-\mathrm{I}_{h}^{2} u^{2}\right\|_{0} & \lesssim h^{s}\left\|u^{2}\right\|_{s,} .
\end{aligned}
$$

Proof. The proof of (83a) goes exactly as the one of (19a), with minor changes. Let $v \in \mathcal{T}_{h}^{0}$ be a vertex on $\partial \Gamma$, and let $N_{v}$ be the number of $t \in \mathcal{T}_{h}^{2}$ which share $v$ as a vertex. We map $v^{\prime}$ into $\widetilde{C}_{N_{v}}=\left\{\widehat{C}_{2 N_{v}}: x<0\right\}$. Let $u^{0} \in \mathrm{H}^{1+s}(\Gamma), \widehat{u}^{0}$ be its pull back on the half cell, $\widehat{\mathrm{I}}_{b}^{0}$ the interpolation operator on $\widetilde{C}_{N_{v}}$ associated with the pull-pack of the boundary basis functions, and $R: \mathrm{H}^{1+s}\left(\widetilde{C}_{N_{v}}\right) \rightarrow \mathrm{H}^{1+s}\left(\widehat{C}_{2 N_{v}}\right)$ be the reflection operator. It is a matter of straightforward checking to see that $\widehat{\mathrm{I}}_{b}^{0} \widehat{u}^{0}=\widehat{\mathrm{I}}^{0} R\left(\widehat{u}^{0}\right)$ on $\widetilde{C}_{N_{v}}$. The estimate 83a) follows directly.

The estimate (83b) follows by the same argument, just noticing that (25) applies as soon as constant vectors are reproduced by the discrete space.

Finally, we prove (83c). Let $u^{2} \in \mathrm{H}^{s}(\Gamma)$. We denote by $\Gamma_{h}$ the total support of the functions in $Y_{h}^{2}$ and note that $\Gamma \backslash \Gamma_{h}$ is a strip of elements of $\mathcal{T}_{h}^{\prime}$ which share with $\partial \Gamma$ an edge or a vertex. A straightforward application of (19c) implies that:

$$
\left\|u^{2}-\mathrm{I}_{h}^{2} u^{2}\right\|_{0} \lesssim h^{s}\|u\|_{s, \Gamma_{h}}+\left\|u^{2}\right\|_{0, \Gamma \backslash \Gamma_{h}}, \quad s \in\left(0, \frac{1}{2}\right) .
$$

It can be proved that for any $v \in \mathrm{H}_{0}^{1}(\Gamma)$ there holds:

$$
\|v\|_{0, \Gamma \backslash \Gamma_{h}} \lesssim h|v|_{1, \Gamma \backslash \Gamma_{h}} .
$$

By standard interpolation theory and recalling that $\mathrm{H}_{0}^{s}(\Gamma)=\mathrm{H}^{s}(\Gamma)$ for any $s \in$ $\left[0, \frac{1}{2}\right)$, the result follows.

The results of Section 3.2. namely Propositions 3.5 and 3.6. are also valid in the case of an open manifold. Indeed, they are consequences of Proposition 4.2 and Theorem 4.3. their statements do not change and their proofs change only in the construction of the continuous Hodge decomposition which is now the one used by Amrouche et al. in 2, Formulae $(3.29,3.30,3.31)]$ In Proposition 3.6, the stability estimate in (37) fails to be true for the singular exponent $t=0$ and extends to the following:

$$
\left\|u_{\perp}^{1}\right\|_{-\frac{1}{2}, \text { curl }, 00}+\left\|u^{0}\right\|_{\frac{1}{2}} \lesssim\left\|u^{1}\right\|_{-\frac{1}{2}, \text { curl }, 00} .
$$

Finally, we arrive at the LBB inf-sup conditions, i.e., the non-degeneracy of the Hodge duality products. Indeed, as before, they are direct consequences of the LBB inf-sup conditions which we have already proved in Section 3.3. Here below, we detail the reasoning.

\footnotetext{
${ }^{2}$ Indeed, the vectorfield $\varphi$ is constructed in both proofs as the unique solution of the problem: $\varphi \in \mathrm{H}_{\operatorname{div}, 0}(\Gamma): \operatorname{curl} \varphi=\operatorname{curl} u^{1} \operatorname{div} \varphi=0$.
} 
$\mathrm{L}^{2}$ duality on $\mathrm{Y}_{h}^{0} \times \mathrm{X}_{h}^{2}$. In the $L^{2}$ context, it is a matter of computing $\int \mu_{s}^{0, b} \lambda_{t}^{2}$ for each pair of triangles $s, t \in \mathcal{T}_{h}$ such that $s \cap \partial \Gamma \in \mathcal{T}_{h}^{1}$. A simple calculation shows that: $\int_{s} \mu_{s}^{0, b} \lambda_{s}^{2}=10 / 18+1 / 9\left(1 / N_{u}+1 / N_{v}+1 / N_{w}\right)$ where $u, v, w$ denote the vertices of $s$. Then, the arguments used in the proof of Proposition 3.9 apply. The arguments used in Proposition 3.10 apply for $s \in[0,1 / 2]$. Recall that, beyond this range, the spaces $H^{s}(\Gamma)^{\prime}$ can no longer be interpreted as spaces of distributions.

$\mathrm{L}^{2}$ duality on $\mathrm{Y}_{h}^{2} \times \mathrm{X}_{h}^{0}$. In the $L^{2}$ context, this is just a special case of the duality proved in Propositions 3.12, Beyond the $L^{2}$ context we use the stability of the $\mathrm{L}^{2}$ projector $R_{h}: \mathrm{H}_{0}^{1}(\Gamma) \rightarrow X_{h}^{0}$ in $\mathrm{H}^{1}$ norm (Crouzeix-Thomée [19]). The exponent $s=\frac{1}{2}$ is singular in the interpolation argument used in Proposition 3.13 . For the case $s=\frac{1}{2}$ the very same interpolation argument provides the following LBB condition: there exists a positive constant $C$ s.t.

$$
\inf _{u \in \mathrm{X}_{h}^{0}} \sup _{v \in \mathrm{Y}_{h}^{2}} \frac{\int u \cdot v}{\|u\|_{\frac{1}{2}, 00}\|v\|_{-\frac{1}{2}, 00}} \geq 1 / C .
$$

$\mathrm{L}^{2}$ duality on $\mathrm{Y}_{h}^{1} \times \mathrm{X}_{h}^{1}$. The proof of Proposition 3.14 relies on the LBB stability of the previous two dualities, and thus it also applies to the present case. More precisely, (68) holds true for all $s \in\left(-\frac{1}{2}, 0\right)$. The cases $s>0$ cannot be considered here since, as already discussed for the duality on $\mathrm{Y}_{h}^{0} \times \mathrm{X}_{h}^{2}$, this would bring us beyond the distributional setting. The case $s=0$ corresponds to the singular index for the duality $\mathrm{Y}_{h}^{2}-\mathrm{X}_{h}^{0}$. The same argument as the one used in the proof of Proposition 3.14, together with (84), provides the following: there exists a constant $C>0$ such that

$$
\inf _{u \in \mathrm{X}_{h}^{1}} \sup _{v \in \mathrm{Y}_{h}^{1}} \frac{\int u \cdot v}{\|u\|_{-\frac{1}{2}, \operatorname{div}}\|v\|_{-\frac{1}{2}, \text { curl }, 00}} \geq 1 / C .
$$

Note that, according to Buffa-Ciarlet [12, pp. 46-47], this is the relevant duality between traces.

4.3. Applications. One of the motivations for constructing the dual spaces of this paper was to obtain spectrally equivalent preconditioners for the Electric Field Integral Equation (EFIE). This equation is routinely solved in electromagnetic scattering problems, with applications to radar cross section computations and antenna simulations. With the advent of Fast Multipole Methods, which enable fast matrixvector multiplication (the complexity is reduced from $O\left(n^{2}\right)$ of full $n \times n$ matrices to $O\left(n \log (n)^{k}\right)$ via diagonalization of the translation operator and a divide and conquer strategy - Rokhlin [25, Coifman et al. [18, and Song et al. 26]) it has become important to design good preconditioners to be incorporated in iterative linear equation solvers.

For the EFIE, variational preconditioners based on the Calderon formulas were first introduced in Christiansen-Nédélec [14. At that time a curl-conforming finite element space in $\mathrm{L}^{2}$ duality with $X_{h}^{1}$ was not known, and the authors resorted to a saddlepoint formulation. The preconditioner thus obtained was not in general invertible, but still several properties could be proved or demonstrated numerically. With the help of the new space $Y_{h}^{1}$ we can obtain a conceptually simpler discretization of the Calderon formulas yielding an invertible preconditioner. This is what we detail in this section. For the rest of it we put $X_{h}=X_{h}^{1}$ and $Y_{h}=Y_{h}^{1}$.

We consider the scattering of an electromagnetic wave by a perfect conductor. More precisely, in $\mathbb{R}^{3}$ we consider an open subset $\Omega$, which is connected and is the 
complement of a (compact) polyhedron with boundary $\Gamma$. We are given an incident wave $\left(E^{i n c}, H^{i n c}\right)$ and we look for the scattered field $(E, H)$. These are vectorfields in $\Omega$ satisfying:

$$
\operatorname{curl} E=+i \omega \mu H \quad \text { et } \quad \operatorname{curl} H=-i \omega \epsilon E,
$$

where $\mu$ is the magnetic permeability, $\epsilon$ is the electric permittivity and $\omega$ the angular frequency of the incident wave. The scattered field also satisfies the Silver-Müller radiation condition at infinity :

$$
\mu^{1 / 2} H \times x /|x|-\epsilon^{1 / 2} E=o(1 /|x|),
$$

and the perfect conductor boundary condition on $\Gamma$ for the total field $E^{\text {tot }}=E+$ $E^{i n c}$ gives (for the tangent components on $\left.\Gamma, E_{T}=(n \times E)_{\mid \Gamma}\right)$ :

$$
E_{\mathrm{T}}=-E_{\mathrm{T}}^{i n c} \text { in } \mathrm{H}_{\mathrm{div}}^{-\frac{1}{2}}(\Gamma) .
$$

The associated wavenumber is $k=\omega(\mu \epsilon)^{1 / 2}$. The Greens functions for the Helmholtz equation is:

$$
G_{k}(x, y)=\frac{e^{i k|x-y|}}{4 \pi|x-y|} .
$$

The scattered electric field can be sought in the form of an electric potential:

$$
E(y)=\left(1+\left(1 / k^{2}\right) \operatorname{grad} \operatorname{div}\right) \int_{\Gamma} G_{k}(x, y) u(x) \mathrm{d} x,
$$

where $u \in \mathrm{H}_{\mathrm{div}}^{-\frac{1}{2}}(\Gamma)$ is an unknown tangent vectorfield on $\Gamma$ determined by the boundary condition 88).

Let $A_{k}$ denote the operator which to $u \in \mathrm{H}_{\text {div }}^{-\frac{1}{2}}(\Gamma)$ associates the tangential trace on $\Gamma$ of the electric potential (90) (i.e., $\left.n \times(E \times n)_{\mid \Gamma}\right)$. It is known that $A_{k}$ is a Fredholm operator $\mathrm{H}_{\text {div }}^{-\frac{1}{2}}(\Gamma) \rightarrow \mathrm{H}_{\text {curl }}^{-\frac{1}{2}}(\Gamma)$ with index 0 and which is invertible for all $k \in \mathbb{R}_{+}$except a countable subset accumulating only at infinity. Let $a$ denote the bilinear form on $\mathrm{H}_{\mathrm{div}}^{-\frac{1}{2}}(\Gamma)$ induced by $A_{k}$ :

$$
a\left(u, u^{\prime}\right)=\left\langle A_{k} u, u^{\prime}\right\rangle .
$$

Recall that:

$$
\begin{aligned}
\left\langle A_{k} u, u^{\prime}\right\rangle= & \iint G_{k}(x, y) u(x) \cdot u^{\prime}(y) \mathrm{d} x \mathrm{~d} y \cdots \\
& -\left(1 / k^{2}\right) \iint G_{k}(x, y) \operatorname{div} u(x) \operatorname{div} u^{\prime}(y) \mathrm{d} x \mathrm{~d} y .
\end{aligned}
$$

Following Bendali [4] one then looks for $u_{h} \in X_{h}$ such that for all $u_{h}^{\prime} \in X_{h}$ :

$$
a\left(u_{h}, u_{h}^{\prime}\right)=-\left\langle E^{i n c}, u_{h}^{\prime}\right\rangle .
$$

Let $A_{h}$ be defined by $\left(A_{h}\right)_{t, s}=a\left(\lambda_{s}^{1}, \lambda_{t}^{1}\right)$ for all $s, t \in \mathcal{T}_{h}^{1}$, be the associated stiffness matrix and $\mathcal{A}_{h}: X_{h} \rightarrow X_{h}^{\star}$ the associated mapping: $\left\langle\mathcal{A}_{h} u_{h}, v_{h}\right\rangle=a\left(u_{h}, v_{h}\right)$. The aim of this section is to construct an optimal preconditioner for the matrix $A_{h}$. Following Christiansen-Nédélec [16] (based on the work by Steinbach-Wendland [27), we need two ingredients:

(i) A uniformly invertible mapping $\mathcal{B}_{h}: Y_{h} \rightarrow X_{h}^{\star}$. Here we choose the mapping associated with the $\mathrm{L}^{2}$ duality. Thus, with our choice of bases, the matrix associated with $\mathcal{B}_{h}$ is $\left(B_{h}\right)_{t, s}=\int \lambda_{t}^{1} \cdot \mu_{s}^{1}$. 
(ii) A uniformly invertible mapping $\mathcal{C}_{h}: Y_{h} \rightarrow Y_{h}^{\star}$. The Calderon formulas suggest one possible choice for $\mathcal{C}_{h}$. Other choices are also possible, as briefly discussed in 14. We denote by $n$ the outward pointing normal on $\Gamma$, and $u \mapsto u \times n$ provides an isomorphism $\Xi: \mathrm{H}_{\text {curl }}^{-\frac{1}{2}}(\Gamma) \rightarrow \mathrm{H}_{\text {div }}^{-\frac{1}{2}}(\Gamma)$. Then, we consider the mapping defined by:

$$
\left\langle\mathcal{C}_{h} v_{h}, v_{h}^{\prime}\right\rangle=a\left(\Xi v_{h}, \Xi v_{h}^{\prime}\right) \quad v_{h}, v_{h}^{\prime} \in Y_{h} .
$$

The fact that $\mathcal{C}_{h}$ is uniformly invertible on $Y_{h}$ (for $h$ small enough) is a consequence of the properties of the complex $Y_{h}^{\bullet}$ proved in this paper (see Section 3.2) and the generalities proved for discretizations of the EFIE in Buffa-Christiansen [9]. We denote by $C_{h}$ the matrix associated with the mapping $\mathcal{C}_{h}$.

The mapping $\mathcal{B}_{h}^{\star-1} \mathcal{C}_{h} \mathcal{B}_{h} \mathcal{A}_{h}$ is the composition of uniformly invertible isomorphisms, thus it is a uniformly invertible isomorphism from $X_{h}$ to $X_{h}$. The general theory now proves that $B_{h}^{\star-1} C_{h} B_{h}^{-1}$ is a preconditioner for $A_{h}$ which is spectrally equivalent in the following sense:

Proposition 4.4. For each $k$ such that the operator $A_{k}: \mathrm{H}_{\mathrm{div}}^{-\frac{1}{2}}(\Gamma) \rightarrow \mathrm{H}_{\mathrm{curl}}^{-\frac{1}{2}}(\Gamma)$ is invertible, the spectral radius of $B_{h}^{\star-1} C_{h} B_{h}^{-1} A_{h}$ and its inverse are both bounded independently of $h$ (for $h$ small enough).

Proof. See $§ 1.2 .2$ in Christiansen [15] and in particular Proposition 1.2.1.

Remark also that $B_{h}^{\star-1} C_{h} B_{h}^{-1} A_{h}$ can be viewed as the matrix of an approximation of the operator $\Xi^{\star} A_{k} \Xi A_{k}$ appearing in the Calderon formulas. This operator is of the form $(1 / 4) I+K$ where, for smooth surfaces, $K: \mathrm{H}_{\text {div }}^{-\frac{1}{2}}(\Gamma) \rightarrow \mathrm{H}_{\mathrm{div}}^{-\frac{1}{2}}(\Gamma)$ is compact.

\section{ApPEndix}

The following proposition is of limited practical interest but shows why we resorted to Helmholtz-Hodge decompositions.

Proposition 5.1. For each pair of edges $s, t \in \mathcal{T}_{h}^{1}$ put $a^{s t}=\int \mu_{s}^{1} \lambda_{t}^{1}$. The matrix $\left(a^{s t}\right)$ is not in general diagonally dominant by rows.

Proof. The computation is based on the fact that there is a constant $\alpha$ such that if any triangle carries a Raviart-Thomas field with coefficients $a, b, c$ according to normals oriented outwards and a Nédélec field with coefficients $a^{\prime}, b^{\prime}, c^{\prime}$ according to counterclockwise oriented tangents, then the integral of their scalar product is $\alpha$ times:

$$
a\left(c^{\prime}-b^{\prime}\right)+b\left(a^{\prime}-c^{\prime}\right)+c\left(b^{\prime}-a^{\prime}\right) .
$$

Using this formula repeatedly gives the following results (where we simplify all matrix coefficients by $\alpha$ ).

Let $s$ be an edge with vertices $u$ and $v$. We get:

$$
a^{s s}=4-\left(1 / N_{u}+1 / N_{v}\right)(8 / 3) \text {. }
$$

Moreover the off-diagonal terms come in several forms (looking at a line in the matrix):

- We have two terms looking like:

$$
a^{s t}=3 / 4+1 /\left(2 N_{u}\right)(-7 / 2)+1 /\left(2 N_{v}\right)(-2 / 3),
$$


and two similar terms obtained by switching the roles of $u$ and $v$.

- We have $N_{u}-3$ terms looking like:

$$
a^{s t}=1 /\left(2 N_{u}\right)(-10 / 3),
$$

and as many obtained by switching the roles of $u$ and $v$.

- We also have $N_{u}-3$ terms of the form:

$$
a^{s t}=i /\left(3 N_{u}\right),
$$

for $i$ ranging between $N_{u}-3$ and $-N_{u}+3$ by steps of 2 . We also have the $N_{v}-3$ terms obtained by substituting $v$ for $u$.

If we add the absolute values of the off-diagonal row terms of the two first types we get:

$$
19 / 3+\left(1 / N_{u}+1 / N_{v}\right)(-55 / 6) ;
$$

those of the third type are best computed by hand. As an example if $N_{u}=N_{v}=6$ we get a diagonal term equal to 56/18 and a sum of absolute values of off-diagonal terms equal to $75 / 18$.

\section{ACKNOWLEDGMENTS}

This work was started while the first author was a visitor at CMA, University of Oslo, May 24-28, 2004, and continued while the second author was a visitor at IMATI, CNR Pavia, October 4-8, 2004. We are grateful to both institutions for their kind hospitality.

\section{REFERENCES}

1. A. Alonso and A. Valli, An optimal domain decomposition preconditioner for low-frequency time-harmonic Maxwell equations, Math. Comput. 68 (1999), No.226, 607-631. MR 1609607 (99i:78002)

2. C. Amrouche, C. Bernardi, M Dauge, and V. Girault, Vector potentials in three-dimensional non-smooth domains, Math. Meth. Appl. Sci. 21 (1998), 823-864. MR.1626990 (99e:35037)

3. Douglas N. Arnold, Differential complexes and numerical stability, Proceedings of the International Congress of Mathematicians, Vol. I (Beijing, 2002) (Beijing), Higher Ed. Press, 2002, pp. 137-157. MR.1989182 (2004h:65115)

4. A. Bendali, Numerical analysis of the exterior boundary value problem for the time-harmonic maxwell equations by a boundary finite element method. I: The continuous problem. II: The discrete problem, Math. Comp. 43 (1984), no. 167, 29-46 and 47-68. MR0744923(86i:65071a). MR0744924 (86i:65071b)

5. A. Bossavit, Mixed finite elements and the complex of Whitney forms, The mathematics of finite elements and applications, VI (Uxbridge, 1987), Academic Press, London, 1988, pp. $137-$ 144. MR956893 (89k:58028)

6. Susanne C. Brenner and L. Ridgway Scott, The mathematical theory of finite element methods, second ed., Texts in Applied Mathematics, vol. 15, Springer-Verlag, New York, 2002. MR 1894376 (2003a:65103)

7. F. Brezzi and M. Fortin, Mixed and hybrid finite element methods, vol. 15, Springer-Verlag, Berlin, 1991. MR 1115205(92d:65187)

8. A. Buffa, Traces theorems for functional spaces related to Maxwell equations: an overview, Computational Electromagnetics (Kiel, Germany) (C. Castersen et al., ed.), Lectures notes in Computational Science and Engineering, vol. 28, Springer-Verlag, 2001.

9. A. Buffa and S. H. Christiansen, The electric field integral equation on Lipschitz screens: definitions and numerical approximation, Numer. Math. 94 (2003), no. 2, 229-267. MR 1974555 (2004c:78016)

10. A dual finite element complex on the barycentric refinement, Comptes Rendus Mathematique 340 (2005), no. 6, 461-464. MR2135331 
11. A. Buffa and P. Ciarlet, Jr., On traces for functional spaces related to Maxwell's equations. Part I: An integration by parts formula in Lipschitz polyhedra, Math. Meth. Appl. Sci. 21 (2001), no. 1, 9-30. MR1809491 (2002b:78024)

12. - On traces for functional spaces related to Maxwell's equations. Part II: Hodge decompositions on the boundary of Lipschitz polyhedra and applications, Math. Meth. Appl. Sci. 21 (2001), no. 1, 31-48. MR1809492 (2002b:78025)

13. A. Buffa, R. Hiptmair, T. von Petersdorff, and C. Schwab, Boundary element methods for Maxwell transmission problems in Lipschitz domains, Numer. Math. 95 (2003), no. 3, 459485. MR2012928(2004i:65131)

14. Snorre H. Christiansen and Jean-Claude Nédélec, A preconditioner for the electric field integral equation based on Calderon formulas, SIAM J. Numer. Anal. 40 (2002), no. 3, 1100-1135 (electronic). MR 1949407(2003m:65235)

15. Snorre Harald Christiansen, Résolution des équations intégrales pour la diffraction d'ondes acoustiques et électromagnétiques, Ph.D. thesis, Ecole Polytechnique, Palaiseau, France, 2001.

16. Snorre Harald Christiansen and Jean-Claude Nédélec, Des préconditionneurs pour la résolution numérique des équations intégrales de frontière de l'acoustique, C. R. Acad. Sci. Paris Sér. I Math. 330 (2000), no. 7, 617-622. MR1760450(2001c:76088)

17. $\mathrm{Ph}$. Ciarlet, The finite element method for elliptic problems, North-Holland, Amsterdam, 1978. MR 0520174 (58:25001)

18. R. Coifman, V. Rokhlin, and S. Wandzura, The fast multipole method for the wave equation: a pedestrian prescription, IEEE Trans. Ant. Prop. 35 (1993), 7-12.

19. M. Crouzeix and V. Thomée, The stability in $L_{p}$ and $W_{p}^{1}$ of the $L_{2}$-projection onto finite element function spaces, Math. Comp. 48 (1987), no. 178, 521-532. MR878688 (88f:41016)

20. Sergei I. Gelfand and Yuri I. Manin, Methods of homological algebra, second ed., Springer Monographs in Mathematics, Springer-Verlag, Berlin, 2003. MR1950475 (2003m:18001)

21. R. Hiptmair and C. Schwab, Natural boundary element methods for the electric field integral equation on polyhedra, SIAM J. Numer. Anal. 40 (2002), no. 1, 66-86 (electronic). MR1921910 (2003j:78059)

22. Roger A. Horn and Charles R. Johnson, Matrix analysis, Cambridge University Press, Cambridge, 1985. MR832183(87e:15001)

23. J.-L. Lions and E. Magenes, Problèmes aux limites non homogènes et applications, Dunod, Paris, 1968.

24. P Monk, Finite Element Methods for Maxwell's Equations, Numerical Mathematics and Scientific Computation, Oxford University Press, 2003. MR2059447 (2005d:65003)

25. V. Rokhlin, Diagonal forms of translation operators for the Helmholtz equation in three dimensions, Appl. Comput. Harmon. Anal. 1 (1993), no. 1, 82-93. MR.1256528 (95d:35034)

26. J. Song, C. Lu, and W.C. Chew, Multilevel fast multipole algorithm for electromagnetic scattering by large complex objects, IEEE Trans. Ant. Prop. 45 (1997), 1488-1493.

27. O. Steinbach and W. L. Wendland, The construction of some efficient preconditioners in the boundary element method, Adv. Comput. Math. 9 (1998), no. 1-2, 191-216. MR 1662766 (99j:65219)

28. Michael E. Taylor, Partial differential equations, Texts in Applied Mathematics, vol. 23, Springer-Verlag, New York, 1996, Basic theory. MR1395147(98b:35002a)

Istituto di Matematica Applicata e Tecnologie Informatiche - CNR, Via Ferrata 1, 27100 Pavia, Italy

E-mail address: annalisa@imati.cnr.it

CMA c/o Matematisk Institutt, PB 1053 Blindern, Universitetet i Oslo, NO-0316 OSLO, NORWAY

E-mail address: snorrec@math.uio.no 